\title{
LncRNA LINRIS stabilizes IGF2BP2 and promotes the aerobic glycolysis in colorectal cancer
}

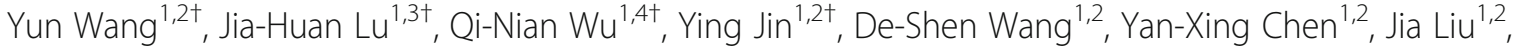

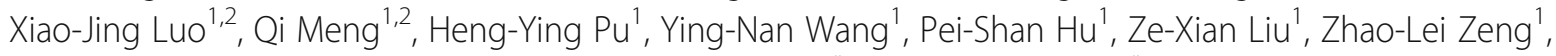 \\ Qi Zhao ${ }^{1}$, Rong Deng ${ }^{1}$, Xiao-Feng Zhu ${ }^{1}$, Huai-Qiang Ju $u^{1,5^{*}}$ and Rui-Hua $X^{1,2,5^{*}}$ (D
}

\begin{abstract}
Background: Long noncoding RNAs (InCRNAs) play nonnegligible roles in the epigenetic regulation of cancer cells. This study aimed to identify a specific IncRNA that promotes the colorectal cancer (CRC) progression and could be a potential therapeutic target.

Methods: We screened highly expressed IncRNAs in human CRC samples compared with their matched adjacent normal tissues. The proteins that interact with LINRIS (Long Intergenic Noncoding RNA for IGF2BP2 Stability) were confirmed by RNA pull-down and RNA immunoprecipitation (RIP) assays. The proliferation and metabolic alteration of CRC cells with LINRIS inhibited were tested in vitro and in vivo.

Results: LINRIS was upregulated in CRC tissues from patients with poor overall survival (OS), and LINRIS inhibition led to the impaired CRC cell line growth. Moreover, knockdown of LINRIS resulted in a decreased level of insulin-like growth factor 2 mRNA-binding protein 2 (IGF2BP2), a newly found $N^{6}$-methyladenosine $\left(\mathrm{m}^{6} \mathrm{~A}\right)$ 'reader'. LINRIS blocked K139 ubiquitination of IGF2BP2, maintaining its stability. This process prevented the degradation of IGF2BP2 through the autophagy-lysosome pathway (ALP). Therefore, knockdown of LINRIS attenuated the downstream effects of IGF2BP2, especially MYC-mediated glycolysis in CRC cells. In addition, the transcription of LINRIS could be inhibited by GATA3 in CRC cells. In vivo experiments showed that the inhibition of LINRIS suppressed the proliferation of tumors in orthotopic models and in patient-derived xenograft (PDX) models.
\end{abstract}

Conclusion: LINRIS is an independent prognostic biomarker for CRC. The LINRIS-IGF2BP2-MYC axis promotes the progression of CRC and is a promising therapeutic target.

Keywords: Autophagy, CRC, IGF2BP2, LINRIS, MYC

\section{Background}

Colorectal cancer (CRC) is an aggressive primary intestinal malignancy with the third leading incidence and second highest mortality of all types of cancers worldwide [1]. In China, over 380,000 new cancer cases are projected to be discovered in the colon and rectum

\footnotetext{
* Correspondence: juhq@sysucc.org.cn; xurh@sysucc.org.cn

†Yun Wang, Jia-Huan Lu, Qi-Nian Wu and Ying Jin contributed equally to this work.

${ }^{1}$ State Key Laboratory of Oncology in South China, Collaborative Innovation Center for Cancer Medicine, Sun Yat-sen University Cancer Center, Guangzhou, China

Full list of author information is available at the end of the article
}

annually [2]. Therefore, finding new therapeutic strategies for CRC is of great significance.

Long noncoding RNAs (lncRNAs) are special RNA molecules that are longer than 200 nucleotides long and have no protein-coding potential [3]. As epigenetic regulators in various diseases, including cancers, lncRNAs are involved in biological processes with diverse mechanisms, such as mediating interactions between DNA and proteins, adsorbing microRNAs, and binding to proteins as decoys [4-6]. Accumulating evidence has shown that, just as oncogenes affect the prognosis of patients, some lncRNAs influence the progression and death of cancer

(C) The Author(s). 2019 Open Access This article is distributed under the terms of the Creative Commons Attribution 4.0 International License (http://creativecommons.org/licenses/by/4.0/), which permits unrestricted use, distribution, and 


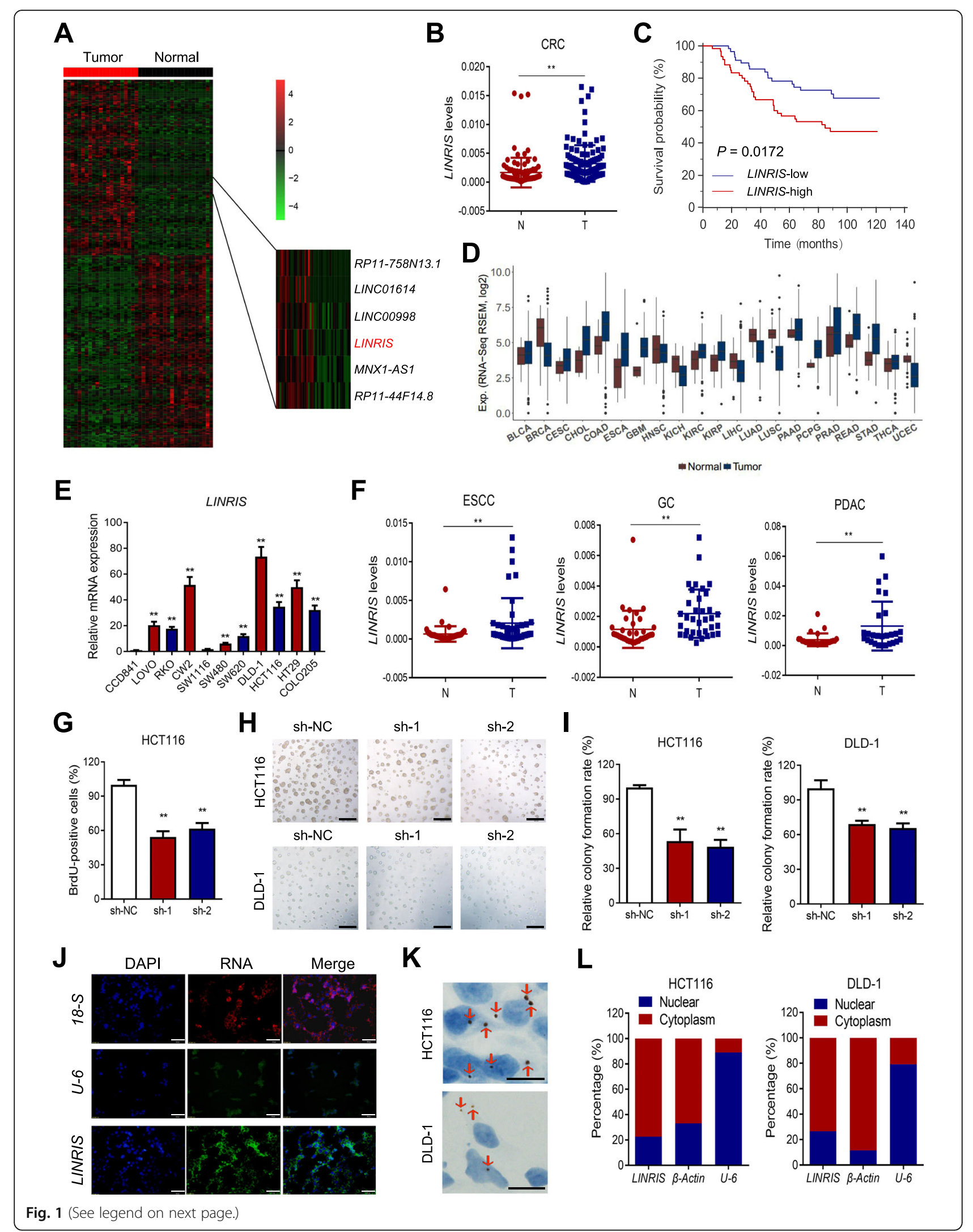


(See figure on previous page.)

Fig. 1 Identification of LINRIS as an oncogenic IncRNA in CRC. a Heatmap for differentially expressed IncRNAs in CRC tissues compared with that in normal tissues. $\mathbf{b}$ qPCR detection shows that LINRIS was highly expressed in CRC tissues $(T, n=118)$ compared with the expression in normal colon tissues (N). ${ }^{*} P<0.05$, ${ }^{* * P}<0.01$. c Kaplan-Meier analysis of the OS curves for CRC patients with LINRIS-low $(n=58)$ or LINRIS-high $(n=60)$ expression (log-rank test). $\mathbf{d}$ The overall LINRIS expression in multiple human cancers from TCGA. e LINRIS levels in different CRC cell lines compared with the level in the normal colon cell line CCD841. The data are shown as the mean $\pm S D ; n=3$ independent experiments, two-tailed Student's t-test, ${ }^{*} P<0.05$, ${ }^{*} P<0.01$. $\mathbf{f}$ qPCR detection shows that LINRIS was highly expressed in ESCC $(n=42)$, GC tissues $(n=35)$ and PDAC $(n=27)$ tissues compared with the expression in normal esophageal, gastric and pancreatic tissues respectively. ${ }^{*} P<0.05,{ }^{*} P<0.01$. g BrdU assays of the indicated cells with LINRIS knockdown by shRNAs compared with the control. The data are shown as the mean \pm SD; $n=3$ independent experiments, two-tailed Student's t-test, ${ }^{*} P<0.05,{ }^{* *} P<0.01$. $\mathbf{h}$ and $\mathbf{i}$ Images $(\mathbf{h})$ and quantification (i) of the 3D culture of the indicated cells with or without knocking down LINRIS after 1 week. Scale bar, $100 \mu \mathrm{m}$. The data are shown as the mean \pm SD; $\mathrm{n}=3$ independent experiments, two-tailed Student's t-test, ${ }^{*} P<0.05,{ }^{*} P<0.01$. j FISH assays identifying the subcellular location of LINRIS in HCT116 cells. Scale bar, $100 \mu \mathrm{m}$. k RNAScope ${ }^{\oplus}$ ISH detection of LINRIS expression (arrows) in CRC cells. Scare bar: $20 \mu \mathrm{m}$. I Location of LINRIS in the cytoplasmic and nuclear extractions from CRC cells with qPCR

cells [7-9], indicating that targeting lncRNAs could be a new approach for CRC treatment.

As another critical epigenetic regulator, the $\mathrm{N}^{6}$ methyladenosine $\left(\mathrm{m}^{6} \mathrm{~A}\right)$ modification has attracted the attention of researchers worldwide [10]. During the biological processes of $\mathrm{m}^{6} \mathrm{~A}$ modifications, there are three types of proteins ('readers', 'writers' and 'erasers') that play irreplaceable roles [11-13]. YT521$\mathrm{B}$ homology domain-containing proteins (YTHDFs) are the well-known $\mathrm{m}^{6} \mathrm{~A}$ 'readers' that participate in the recognition of $\mathrm{m}^{6} \mathrm{~A}$-modified mRNAs [13, 14]. The insulin-like growth factor 2 mRNA-binding protein (IGF2BP) family consists of three members, IGF2BP1-3, which are newly reported $\mathrm{m}^{6} \mathrm{~A}$ 'readers' [14]. Unlike YTHDFs, which regulate pre-mRNA splicing and facilitate translation, these proteins are responsible for targeted mRNA stability and are associated with thousands of targets, such as $M Y C$, KRAS and MDR1 [15-17]. In brief, IGF2BPs recognize $\mathrm{m}^{6} \mathrm{~A}$-modified $\mathrm{mRNAs}$ and maintain their stability by recruiting RNA stabilizers to promote the progression of cancers $[14,18]$. However, the biological mechanism of IGF2BP2 in CRC remains largely unclear.

In this study, we found a highly expressed lncRNA called LINRIS (Long Intergenic Noncoding RNA for IGF2BP2 Stability) in CRC. LINRIS blocked the degradation of IGF2BP2 through the ubiquitination-autophagy pathway. As a consequence, MYC-mediated glycolysis was downregulated, inhibiting the proliferation of CRC cells in vitro and in vivo.

\section{Methods}

\section{Cell lines and cell culture}

All human CRC cell lines described in this article were purchased from the American Type Culture Collection (Manassas, VA, USA). The cells were grown in basic RPMI-1640 medium $(1 \times)$ or DMEM (Thermo Fisher Scientific, Waltham, MA, USA) supplemented with $10 \%$ fetal bovine serum at $37^{\circ} \mathrm{C}$ with $5 \% \mathrm{CO}_{2}$. All cells tested negative for mycoplasma contamination, and this result was verified by short tandem repeat fingerprinting before use.

\section{Reagents and antibodies}

The reagents and antibodies are listed in Additional file 1: Table S1.

\section{RNA-sequencing (RNA-seq) analysis}

With the raw reads from sequencing, we first retained the qualified reads (also known as clean reads) that passed the quality control step by FastQC software. Reads with low base quality, contamination or containing more than $10 \% \mathrm{~N}$ were removed from further analysis. By using STAR [19], clean reads of each sample were then aligned to the GRCh38 human reference genome from GENCODE. Gene and transcript expression were sequentially estimated with RSEM [20]. To compare the lncRNAs, the genes were grouped in terms of "lncRNA", "non_coding" and "antisense" according to the annotation from GENCODE. Differential expression analysis was performed using DESeq2 [21], and those RNAs with an adjusted $P$ value $<0.05$ and a fold change $>1.5$ were considered differentially expressed genes. In addition, the genes with $<1$ fragments per kilobase of transcript per million fragments mapped were removed.

\section{Lentivirus and plasmid transfection}

The expression of LINRIS was knocked down by short hairpin RNAs (shRNAs) targeting human LINRIS or by a nonspecific oligonucleotide that was ligated into the LV-3 (pGLVH1/GFP + Puro) vector. The lentiviruses were synthesized by $\mathrm{OBiO}$ Technology Co., Ltd. (Shanghai, China) and the sequences are listed in Additional file 2: Table S2. HCT116 and DLD-1 cells were transfected with the lentivirus according to the manufacturer's instructions. To obtain stably transfected cell lines, these cells were treated with puromycin $(2-3 \mu \mathrm{g} / \mathrm{mL})$ for 2 weeks. After the knockdown efficiency was confirmed by quantitative PCR (qPCR) 


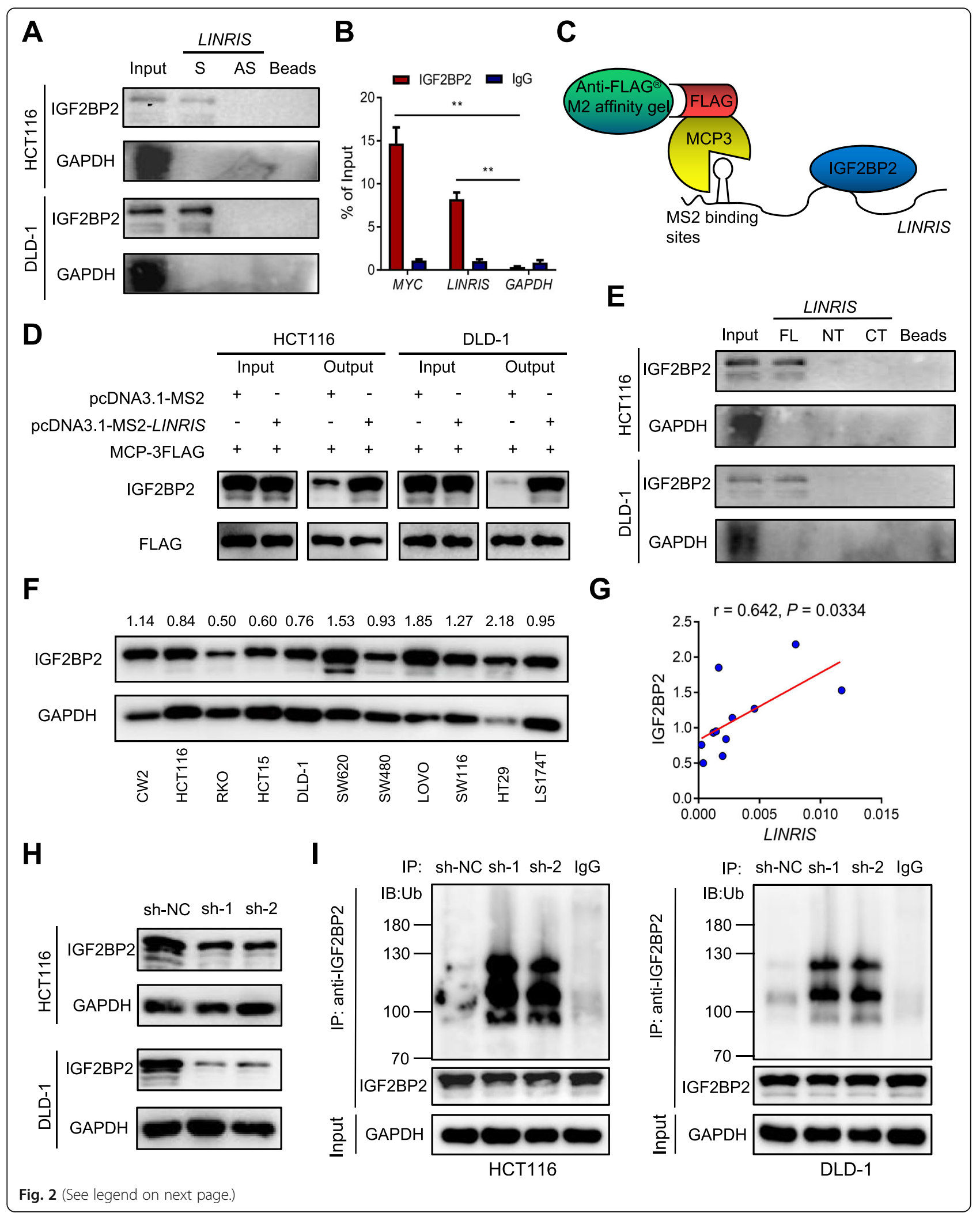


(See figure on previous page.)

Fig. 2 LINRIS was associated with IGF2BP2 in CRC. a IGF2BP2 was pulled down by biotin-labeled sense LINRIS (S) but not LINRIS anti-sense (AS) RNA in the indicated cells. b RIP assays were applied using anti-IGF2BP2 antibodies with extractions from HCT1 16 cells. Relative enrichment (mean \pm SD) represents the RNA levels associated with the indicated protein relative to an input control from three independent experiments after immunoprecipitation with the anti-IGF2BP2 antibody compared with that with the IgG antibody. MYC MRNA was uesd as the positive control and GAPDH mRNA was used as the negative control. c Expression vectors for FLAG-tagged MCP and MS2-tagged LINRIS were transfected into CRC cells to establish the FLAG-MCP-MS2 system. And IGF2BP2 was then pulled down using the anti-FLAG ${ }^{\oplus}$ M2 affinity gel followed by the Western blot analysis. d Western blot detection of IGF2BP2 binding to LINRIS after FLAG-MCP-MS2 pull-down assays. e In vitro-synthesized full-length (FL), N-terminal (NT) and C-terminal (CT) fragments of LINRIS were incubated with protein lysates from HCT116 cells. RNA pull-down and Western blotting assays were then performed. The data shown represent three independent experiments. $\mathbf{f}$ Western blot analysis shows the levels of IGF2BP2 in 11 CRC cell lines with GAPDH as the loading control. $\mathbf{g}$ IGF2BP2 expression was positively correlated with LINRIS expression in CRC cells. The $r$ values and $P$ values are from Pearson's correlation analysis. $\mathbf{h}$ Western blot analysis shows the expression of IGF2BP2 with or without knockdown of LINRIS in the indicated cells. $\mathbf{i}$ CRC cells transfected with shRNAs specific for LINRIS or a scrambled control. Cell lysates were immunoprecipitated with either an antibody against IGF2BP2 or an lgG control and then analyzed by immunoblotting with a ubiquitin (Ub)-specific antibody

and Western blotting analyses, the cells were used for subsequent experiments.

The expression vectors for 3FLAG-tagged MS2 coat protein (MCP) and MS2-tagged LINRIS were provided by $\mathrm{OBiO}$ Technology Co., Ltd. (Shanghai, China), and FLAG-tagged expression vectors for full-length IGF2BP2 and site-directed mutants (K77R and K139R) were provided by Kidan BioTechnology Co., Ltd. (Guangzhou, China). The plasmids were transfected into the cells with Lipofectamine 3000 as recommended by the manufacturer.

\section{Human tissue specimens}

Clinical samples were collected from Sun Yat-sen University Cancer Center (Guangzhou, China). All patients were histologically diagnosed with CRC before the operation. Written informed consent was obtained from all patients. The study was approved by the Medical Ethics Committee of Sun Yat-sen University.

\section{Immunoprecipitation (IP) assay}

An anti-IGF2BP2 antibody (1-2 mg per test, Abcam, ab124930) and an anti-FLAG/DYKDDDDK Tag (1-2 mg per test, Cell Signaling Technology, $8146 \mathrm{~s}$ ) were used in the IP assays, and the proteins were detected by Western blotting with an anti-ubiquitin antibody (1:1000, Cell Signaling Technology, \#3933) according to the manufacturer's instructions.

\section{RNA pull-down and RNA immunoprecipitation (RIP) assays}

Expression vectors for full-length LINRIS and its $\mathrm{N}$ terminal (1-570 nt) and C-terminal (571-913 nt) regions used for the in vitro synthesis of RNA were provided by OBiO Technology (Shanghai, China). The lncRNAs were transcribed in vitro using a MEGAscript $^{\mathrm{TM}}$ T7 Transcription Kit (Invitrogen, Carlsbad, CA, USA) and were biotinylated with a Pierce RNA 3' End Desthiobiotinylation Kit (Thermo
Fisher Scientific, Waltham, MA, USA) according to the manufacturer's instructions. The proteins were extracted from HCT116 and DLD-1 cell lines using Pierce IP Lysis Buffer. Then, RNA pull-down assays were performed with a Pierce Magnetic RNA-Protein Pull-Down Kit (Thermo Fisher Scientific, Waltham, MA, USA). Briefly, the biotinylated IncRNAs were captured with streptavidin magnetic beads and incubated with the cell lysates at $4{ }^{\circ} \mathrm{C}$ for $6 \mathrm{~h}$. Then, the mixture was washed and eluted. The eluate was subjected to mass spectrometry or Western blotting analysis. RIP assays were performed with a Magna RNA-Binding Protein Immunoprecipitation Kit (Millipore, Bedford, MA, USA) according to the manufacturer's instructions. The mixture was digested with proteinase $\mathrm{K}$ before the immunoprecipitated RNAs were extracted, purified and subjected to qPCR. The RNA levels were normalized to the input RNA levels (10\%).

\section{Extracellular acidification rate (ECAR) and the measurement of intracellular metabolites}

ECAR was measured according to the XF Glycolysis Stress Test protocol on a Seahorse XFe24 Extracellular Flux Analyzer (Agilent Technologies, Santa Clara, CA, USA), and ${ }^{13} \mathrm{C}$-labeled intracellular metabolites were identified as previously described [22]. CRC cells (approximately $1 \times 10^{7}$ cells) were incubated with $2 \mathrm{~g} / \mathrm{L}{ }^{13} \mathrm{C}$ labeled glucose for $2 \mathrm{~h}$. Metabolites were extracted and detected with a liquid chromatography system equipped with a TripleTOF 5600 mass spectrometer (SCIEX, Framingham, MA, USA). The concentration of the ${ }^{13} \mathrm{C}$-labeled metabolites was normalized to the cell number.

\section{Chromatin immunoprecipitation (ChIP) assays}

ChIP assays were performed with a ChIP kit from Merck Millipore (Billerica, MA, USA) according to the manufacturer's instructions. qPCR analysis was performed to detect the DNA fragments that coimmunoprecipitated with GATA3. 
A

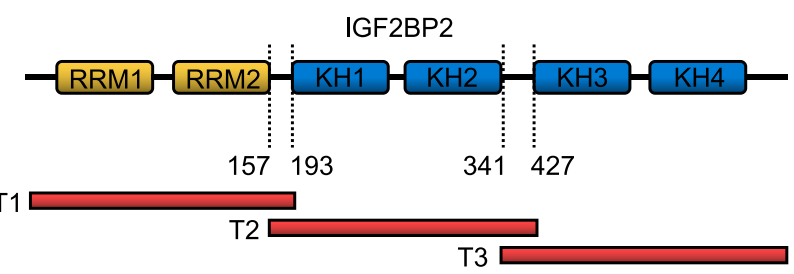

C

IP: $\frac{\text { Sh-1 }}{\text { WT K77R K139R }}$

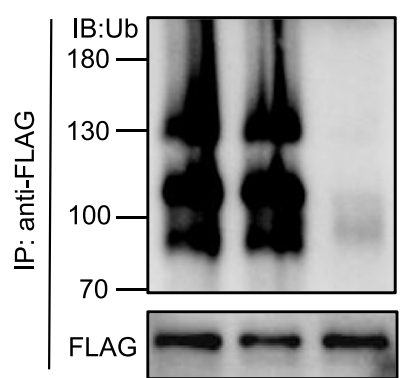

D
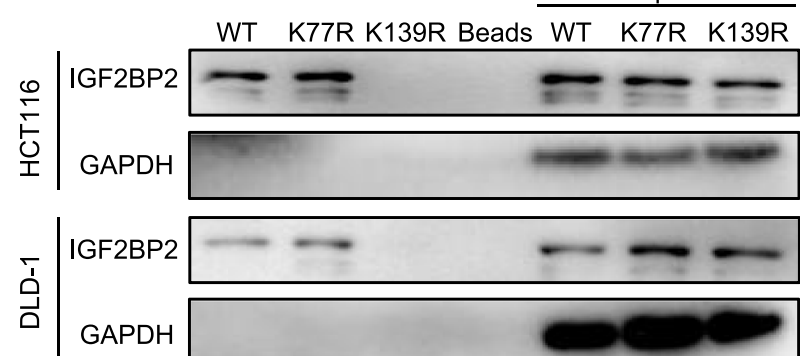

G

(Ub

(Ub) (Ub)

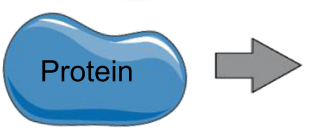

E

E $\mathrm{HCT116}$ sh-NC

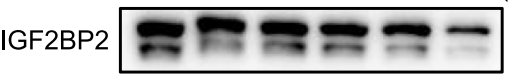

GAPDH

sh-1

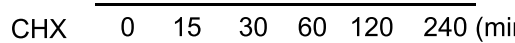

IGF2BP2 $=-\infty \cdots$

GAPDH

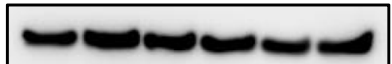

B

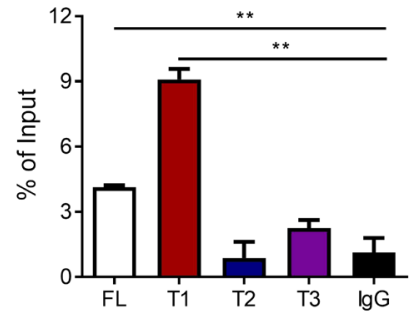

DLD-1 sh-NC

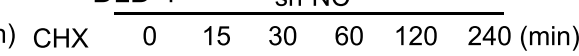

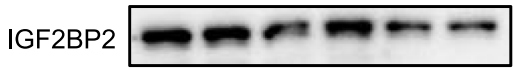

GAPDH

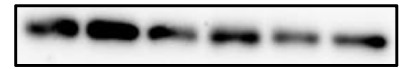

sh-1

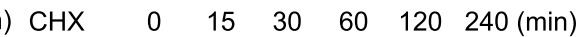

IGF2BP2 $m \omega m+\infty$

GAPDH

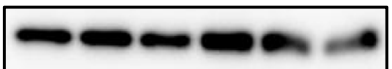

F MG132

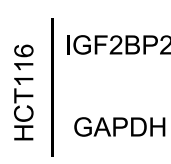

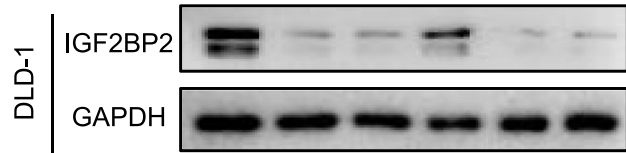

sh-NC sh-1 sh-2 sh-NC sh-1 sh-2

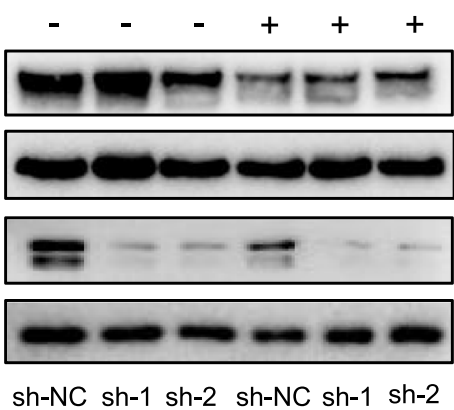

$J$

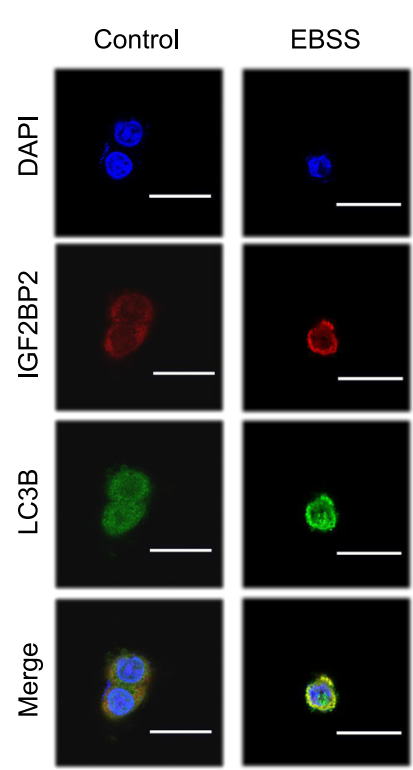

Fig. 3 (See legend on next page.) 
(See figure on previous page.)

Fig. 3 LINRIS is involved in the autophagic degradation of IGF2BP2. a Schematic structures of IGF2BP2 proteins and three truncated mutants (T1: 1193; T2: 157-427; T3: 341-913) of IGF2BP variants used in this study. Orange boxes are RRM domains, and blue boxes are KH domains. b RIP assays were performed using anti-FLAG antibodies and HCT116 cells transfected with vectors expressing the FLAG-tagged FL and the truncated mutants (T1T3) of IGF2BP2. The data shown represent three independent experiments. The data are shown as the mean \pm SD; $n=3$ independent experiments, two-tailed Student's t-test, ${ }^{*} P<0.05,{ }^{* *} P<0.01$. c IP assays were performed using anti-FLAG antibodies and HCT1 16 cells transfected with vectors expressing the FLAG-tagged WT or IGF2BP2 mutants (K77R and K139R). $\mathbf{d}$ Western blot analysis following RNA pull-down assays shows that the K139R mutation of IGF2BP2 blocked its binding to LINRIS in CRC cells. GAPDH was used as the loading control. e CRC cells with or without shRNAs specific for LINRIS were treated with $20 \mathrm{\mu g} / \mathrm{ml}$ CHX or a vehicle for the indicated periods of time. IGF2BP2 levels were analyzed by western blotting. f CRC cells with LINRIS knockdown and control cells were treated with or without MG132 $(5 \mu \mathrm{M})$ for $12 \mathrm{~h}$. Cell lysates were analyzed by Western blotting with GAPDH as the loading control. $\mathbf{g}$ Illustration of the protein degradation process via two main pathways. $\mathbf{h}$ CRC cells with LINRIS knockdown were treated with or without Baf $\mathrm{A} 1(100 \mathrm{nM}, 24 \mathrm{~h})$ and $\mathrm{NH}_{4} \mathrm{Cl}(10 \mathrm{mM}, 4 \mathrm{~h})$. Cell lysates were analyzed by Western blotting with GAPDH as the loading control. i Western blotting shows the levels of IGF2BP2 in the indicated cells after treatment with or without EBSS for 3-6 h or Rap (100 nM) for $24 \mathrm{~h}$. GAPDH was used as the loading control. $\mathbf{j}$ Confocal microscopy of HCT1 16 cells treated with or without EBSS for $3 \mathrm{~h}$. Scale bar, $50 \mu \mathrm{m}$

\section{RNA interference (RNAi)}

The small interfering RNAs (siRNAs) used for in vivo treatment were provided by RiboBio (Guangzhou, China) according to the same sequences as the sh-LINRIS (sh-1 and sh-2). The resulting constructs were verified by sequencing. siRNAs were injected into the tumors at two or more spots each time.

\section{In vivo therapeutic study}

All female BALB/c nude mice (3-4 weeks old) used in our study were purchased from the Beijing Vital River Laboratory Animal Technology Co., Ltd. and then housed in specific pathogen-free units.

For the orthotopic models, $2 \times 10^{6}$ cells with negative control (NC, sh-NC), sh-1 or sh-2 in $0.5 \mathrm{~mL}$ of PBS were subcutaneously injected into the dorsal flank of 2 mice respectively. After the tumors grew up to $1 \mathrm{~cm}^{3}$, they were resected and equally divided into small pieces. Then 15 mice were separated into 3 groups (sh-NC, sh1 and sh-2), of which the tumor pieces were tied to the base of the ceca. The growth of the tumors was monitored every 2 weeks after intraperitoneal injection of D-luciferin with a Xenogen IVIS 100 Bioluminescent Imaging System. All mice were sacrificed 4 weeks after the surgery.

For the PDX models (PDX\#1-3), the tumor tissues were obtained from patients receiving surgeries at our cancer center [23]. After taking a biopsy of a small part of the tumor, we conserved the tissue in ice cold culture medium with $5 \%$ penicillin and streptomycin. The tissue was separated into several pieces, which were implanted into the dorsal flanks of mice. To investigate the antitumor effects of RNAi in each PDX model (\#1 and \#2), 15 female $B A B L / c$ nude mice were randomly assigned into 3 groups (NC, si-LINRIS\# 1 and \#2) 2 weeks later. The RNAi solution (20 $\mathrm{nmol})$ was injected directly into the tumor bodies of si-LINRIS\#1 and \#2 groups twice per week. PDX\#3 was used to investigate the combination effect of RNAi and oxaliplatin. Twenty female BALB/c nude mice were randomly assigned into the following 4 groups: control, oxaliplatin (injected into the abdominal cavity, $5 \mathrm{mg} / \mathrm{kg}$ twice per week), RNAi (injected directly into the tumor bodies, $20 \mathrm{nmol}$ twice per week), or combined treatment. The tumor volumes were recorded twice weekly. After treatment for approximately 4 weeks, the mice were sacrificed and all the tumors were extracted and weighed. Furthermore, the tumor tissues were embedded in paraffin, sectioned and stained with hematoxylin and eosin (H\&E) or immunohistochemically (IHC) stained with antibodies against Ki-67, IGF2BP2 and MYC according to previously reported protocols [24]. Apoptotic cells in situ were also identified by using a Cell Death Detection Kit (Biotool, Houston, TX, USA) for TdTmediated dUTP nick end labeling (TUNEL) staining according to the manufacturer's instructions. The animal study was approved by the Institutional Animal Care and Use Committee of Sun Yat-sen University.

\section{Statistical analysis}

All data are presented as the mean \pm SD. Student's paired or unpaired t-tests and chi-square tests were used for the comparison of significant differences between groups with GraphPad Prism software. Correlations between the LINRIS levels and MYC, GLUT-1, PKM2 and LDHA expression were analyzed with Pearson's correlation analysis. Survival analyses were performed using the Kaplan-Meier method and assessed using the log-rank test with SPSS and MedCalc statistical software. The levels of significance were set at $*$, representing $P<0.05$ and ${ }^{* *}$, representing $P<0.01$.

Additional methods are described in Additional file 3.

\section{Results}

\section{LINRIS is highly expressed in CRC with poor prognosis}

To exploit lincRNAs that potentially influence the progression of CRC, 21 tumor tissues from patients with stage IV CRC were sent for transcriptome/ 
A

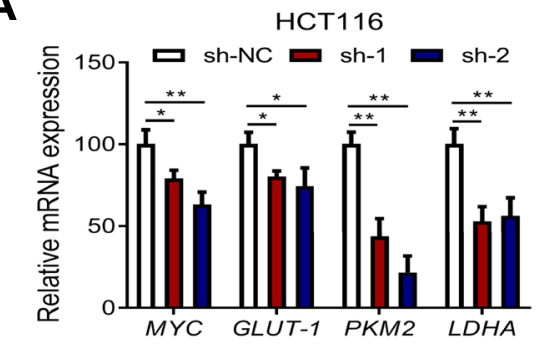

B

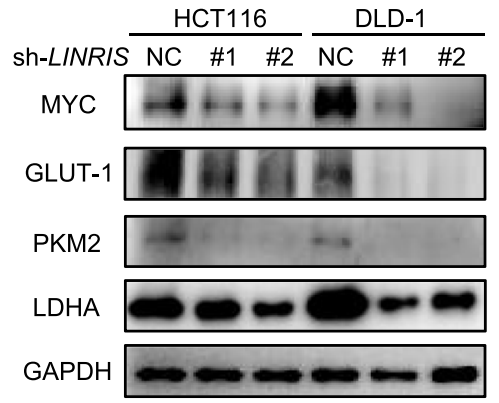

D
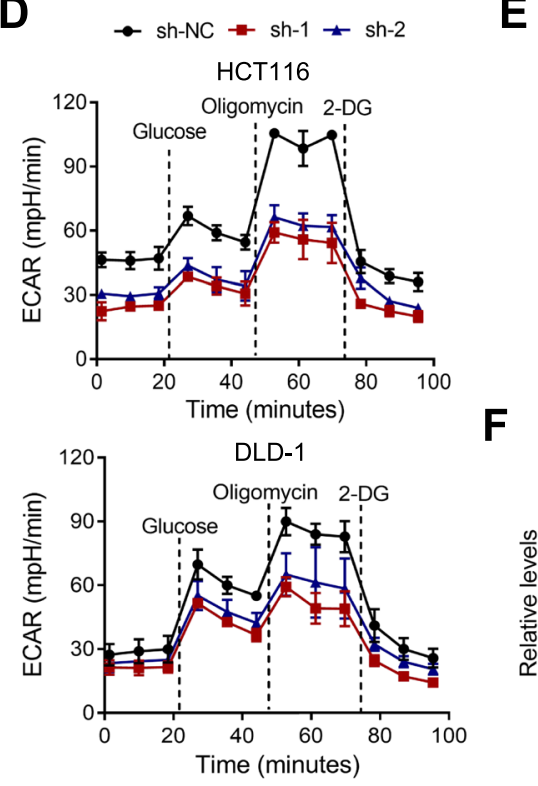

G

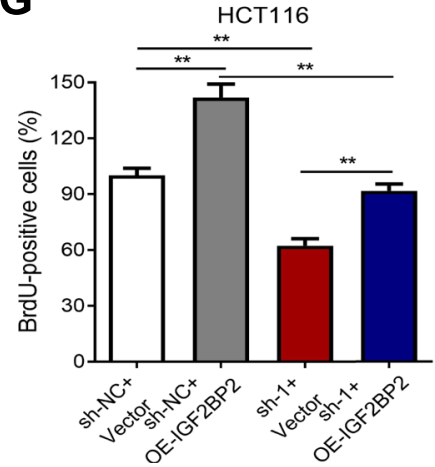

H
C

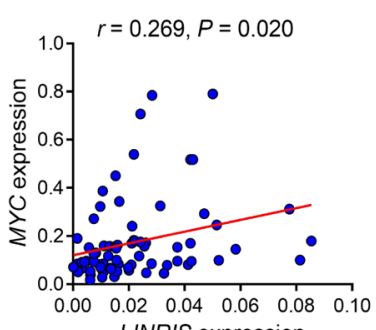

LINRIS expression
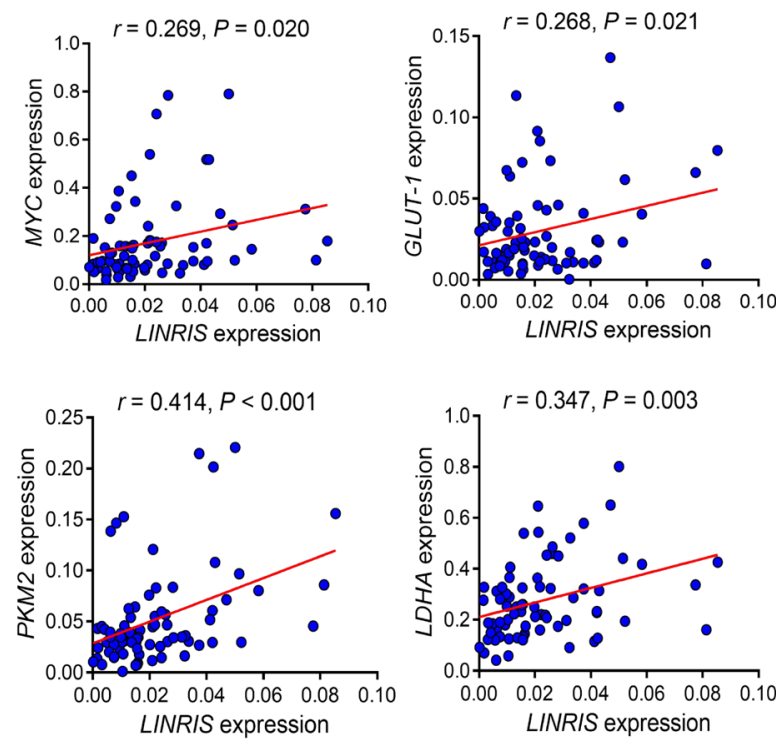

HCT116

DLD-1
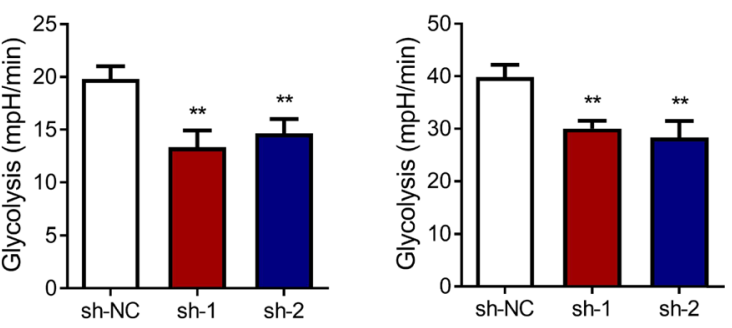

F ${ }^{13} \mathrm{C}$ labelled $3 \mathrm{PG} / 2 \mathrm{PG}$
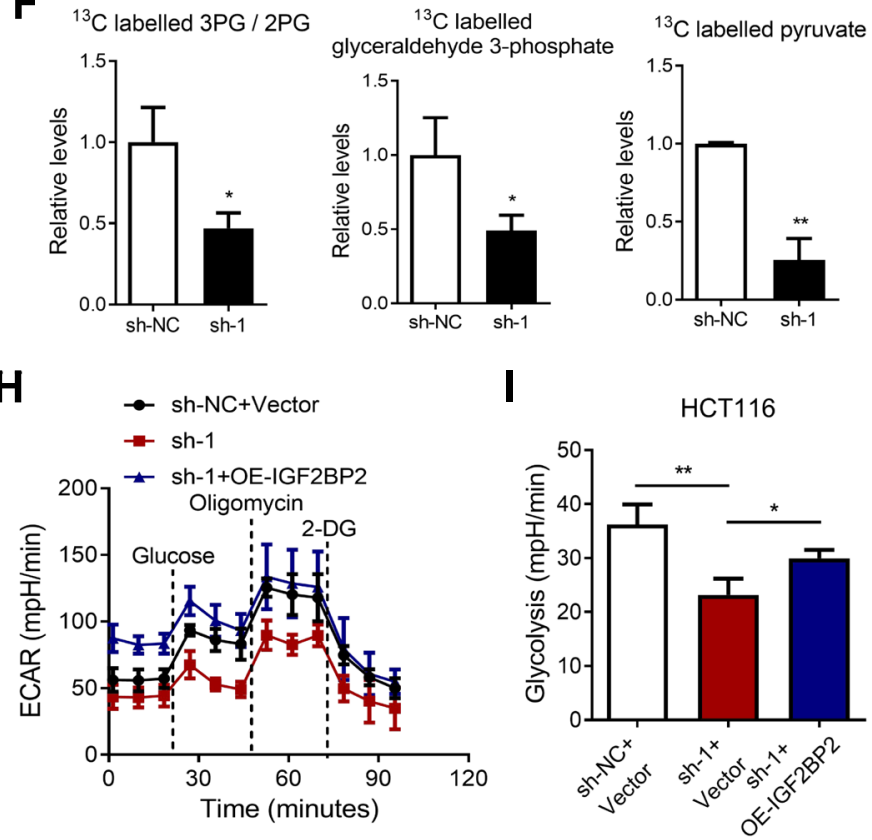

Fig. 4 (See legend on next page.) 
(See figure on previous page.)

Fig. 4 The alteration of MYC-mediated glycolysis under the influence of LINRIS. a The mRNA levels of MYC and downstream genes, including GLUT-1, PKM2 and LDHA, when inhibiting LINRIS in HCT116 cells. The data are shown as the mean \pm SD; $\mathrm{n}=3$ independent experiments, twotailed Student's t-test, ${ }^{*} P<0.05,{ }^{*} P<0.01$. b Western blot analysis showed the levels of MYC and downstream genes, including GLUT-1, PKM2 and LDHA, when inhibiting LINRIS in CRC cells. c Correlations between the LINRIS levels and the mRNA levels of MYC, GLUT-1, HK2, PKM2 and LDHA in CRC $(n=74)$. RNA levels were determined by qPCR relative to the levels of GAPDH. The $r$ values and $P$ values are from Pearson's correlation analysis. $\mathbf{d}$ The ECAR was detected in the indicated cells with sh-1, sh-2 or control using an XF Extracellular Flux Analyzer. Glucose, oligomycin and 2-DG were injected sequentially at different time points as indicated. The data shown represent three independent experiments. e Statistical analysis of the effects of LINRIS knockdown on glycolytic activity. The data are shown as the mean \pm SD; $n=3$ independent experiments, two-tailed Student's t-test, ${ }^{*} P<0.05$, ${ }^{*} P<0.01$. f Statistical analysis of ${ }^{13} \mathrm{C}$-labeled $3-\mathrm{PG} / 2-\mathrm{PG},{ }^{13} \mathrm{C}$-labeled glyceraldehyde 3 phosphate and ${ }^{13} \mathrm{C}$-labeled pyruvate. The data are shown as the mean \pm SD; $\mathrm{n}=3$ independent experiments, two-tailed Student's t-test, ${ }^{*} P<0.05$. $\mathbf{g}$ BrdU assay showing that the overexpression (OE) of IGF2BP2 rescued the proliferation inhibition of the indicated cells with the knockdown of LINRIS. The data are shown as the mean \pm SD; $\mathrm{n}=3$ independent experiments, two-tailed Student's t-test, ${ }^{*} P<0.05,{ }^{*} P<0.01$. $\mathbf{h}$ The extracellular acidification rate (ECAR) was detected in HCT116 cells with or without sh-1 and overexpressed IGF2BP2 using an XF Extracellular Flux Analyzer. Glucose, oligomycin and 2-DG were injected sequentially at different time points as indicated. The data shown represent three independent experiments. i Overexpression of IGF2BP2 reversed the suppression of LINRIS knockdown on glycolytic activity in HCT116 cells. The data are shown as the mean $\pm \mathrm{SD} ; n=3$ independent experiments, two-tailed Student's t-test, ${ }^{*} P<0.05,{ }^{* *} P<0.01$

RNA-seq with paired adjacent normal tissues for comparison. Based on the RNA-seq analysis, we screened out 233 genes coding highly expressed (fold change >2) lncRNAs (Fig. 1a and Additional file 4: Figure S1A). According to the $P$ values and the base mean of these genes, we picked up 30 candidates for further confirmation. Finally, 7 highly expressed lncRNAs were screened for prognosis, including LINC00920 (renamed as LINRIS) (Additional file 4: Figure S1B). Moreover, the RNA-seq analysis showed that NR_046242 (913 bp) was the major transcript of LINRIS in CRC (Additional file 4: Figure S1C). We then discovered that higher LINRIS expression was correlated with an unfavorable overall survival (OS) of patients with CRC ( $n=118$, clinicopathological features are listed in Additional file 5: Table S3) from Sun Yat-sen University Cancer Center (Fig. 1b and Fig. 1c). In addition, multivariate analysis showed that LINRIS was also an independent prognostic factor in the patients with CRC (Additional file 6: Table S4).

According to The Cancer Genome Atlas (TCGA) database, LINRIS expression was upregulated in most kinds of tumors and CRC cell lines compared with the expression in normal cells (Fig. 1d and Fig. 1e); these results indicate that LINRIS generally acts as an oncogene. Our samples from patients with esophageal squamous cell carcinoma (ESCC), gastric cancer (GC) and pancreas ductal adenocarcinoma (PDAC) also showed the oncogenic status of LINRIS in digestive cancers (Fig. 1f). Furthermore, by measuring the expression of LINRIS in CRC cell lines and 8 human CRC samples, we found that the increased copy number was account for the upregulation of LINRIS in CRC (Fig. 1e and Additional file 4: Figure S1D-S1F). Subsequently, we decided to use two cell lines (HCT116 and DLD-1) with a relatively high LINRIS copy number for further research.
Next, we knocked down LINRIS in CRC cells with shRNAs (Additional file 4: Figure S1G). BrdU and the 3D-culture assays were used to compare CRC cells transfected with LINRIS-specific shRNAs (sh-1 and sh-2) with the nagative control (sh-NC), and the results identified the oncogenic function of LINRIS in assisting the growth of cancer cells (Fig. 1g, h, i and Additional file 4: Figure S1H). With RNA FISH assays, we found that LINRIS was mainly located in the cytoplasm (Fig. $1 \mathrm{j}$ and Additional file 4: Figure S1I), which was further confirmed by the RNAScope ${ }^{\circ}$ ISH assays and the qPCR analysis of the nuclear and cytosolic extractions (Fig. $1 \mathrm{k}$ and $\mathrm{l}$ ).

\section{LINRIS interacted with IGF2BP2 and maintained its expression}

To identify the molecular mechanism of the LINRISinduced progression of CRC cells, we performed RNA pull-down assays and subsequent mass spectrometry (MS) analysis to explore the proteins that could be potentially associated with LINRIS $[25,26]$. By comparing independent samples of HCT116 cells with control cells, we mainly identified 18 candidates. Based on the protein score, the protein coverage and the exponentially modified protein abundance index (emPAI) from MS identification (Additional file 7: Figure S2A, B and C), IGF2BP2 was selected as our first target instead of the nuclear proteins such as hnRNPL and HIST1H4A, as it is located mainly in the cytoplasm (Additional file 7: Figure S2D). As shown in Fig. 2a-d, LINRIS could directly bind to IGF2BP2. Computational secondary structure analysis of IncRNA was performed with RNAfold (Additional file 7: Figure S2E) [27]. To locate the binding sites of LINRIS targeting IGF2BP2, we first constructed two truncated LINRIS vectors and performed the RNA pull-down assays, as this lncRNA is only $913 \mathrm{bp}$ in length. However, neither 


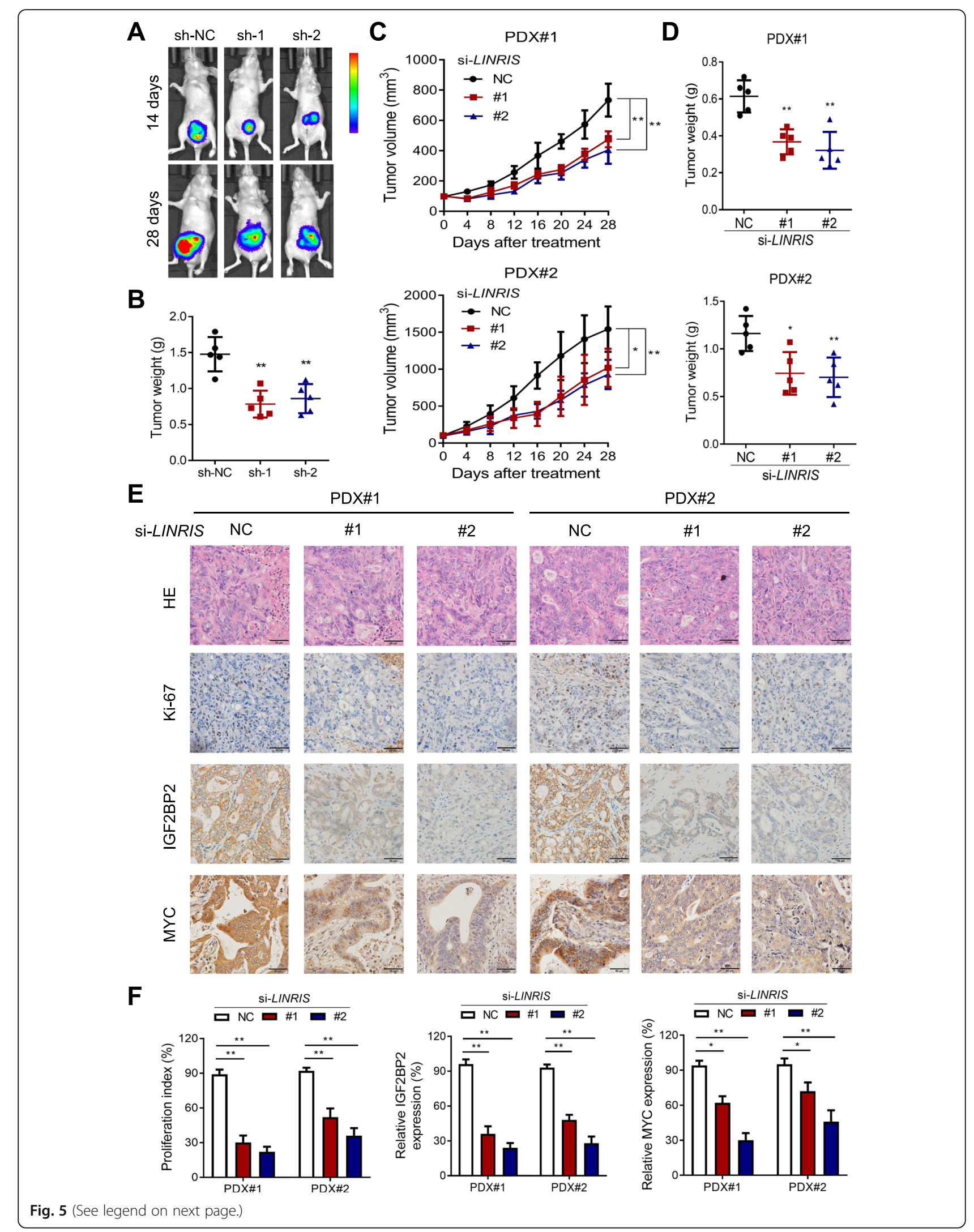


(See figure on previous page.)

Fig. 5 In vivo experiments elucidated the effect of the inhibition of LINRIS in CRC. a Image of orthotopic tumors with or without LINRIS knockdown resected from nude mice. $\mathbf{b}$ The tumor weights of the orthotopic tumors with or without LINRIS knockdown. Error bars, SD of five independent experiments. ${ }^{*} P<0.05$ or ${ }^{* *} P<0.01$ versus the control. $\mathbf{c}$ and $\mathbf{d}$ The volume growth curves of tumors (c) and the tumor weights (d) of two PDX models are shown. Error bars, SD of four independent experiments. ${ }^{*} P<0.05$ or ${ }^{*} P<0.01$ versus the control. e Representative images of H\&E staining and IHC staining of Ki-67, IGF2BP2 and MYC from the tumor sections. Scale bar, $50 \mu m$. $\mathbf{f}$ Quantification of the proliferation index (Ki-67 proportion), IGF2BP2 and MYC levels in the tumor sections. ${ }^{*} P<0.05,{ }^{* *} P<0.01$

the N-terminal (1-570 nt) nor the C-terminal (571$913 \mathrm{nt}$ ) fragments of LINRIS could tightly bind to IGF2BP2 (Fig. 2e). Therefore we constructed another vector with 450-640 nt fragments of LINRIS according to its secondary structure. The RNA pull-down assays showed that this middle region (450-640 nt) was responsible for the binding between LINRIS and IGF2BP2 (Additional file 7: Figure S2F).

By measuring the expression of LINRIS and IGF2BP2 in human CRC cell lines (Fig. 2f and Additional file 7: Figure S2G), we found a positive correlation between them (Fig. 2g). Intriguingly, IGF2BP2 was obviously downregulated with LINRIS knockdown in both HCT116 and DLD-1 cells (Fig. 2h). Moreover, knocking down LINRIS significantly increased the ubiquitination of IGF2BP2 but repressed IGF2BP2regulated mRNAs (Fig. $2 \mathrm{i}$ and Additional file 7: Figure $\mathrm{S} 2 \mathrm{H})$. Therefore, we assumed that IGF2BP2 might be the key to the molecular mechanism of LINRIS and that the degradation of IGF2BP2 was probably prevented by this lncRNA.

\section{LINRIS protected IGF2BP2 from autophagic degradation} IGF2BP2 consists of 2 RNA recognition motifs (RRMs) and $4 \mathrm{~K}$ homology $(\mathrm{KH})$ domains and we established three FLAG-tagged vectors containing fragments of IGF2BP2 overlapping with each other on the inactive sections (Fig. 3a). The RIP assay showed that LINRIS mainly bound to the region of RRMs (1$157 \mathrm{bp)}$ (Fig. 3b). Then, we obtained the experimentally identified ubiquitination sites of IGF2BP2 from the CPLM databases [28], including two ubiquitinated lysine (K) residues (K77 and $\mathrm{K} 139$ ) in this region. As shown in Fig. 3c, the K139R ubiquitin mutation (unable to form K63-linked chains) in place of K77R significantly impaired the ubiquitination of IGF2BP2 compared with that of the wild-type (WT). In addition, this mutation also abolished the binding between LINRIS and IGF2BP2 in RNA pull-down assays (Fig. 3d), suggesting that LINRIS suppressed the ubiquitination of IGF2BP2 by masking K139.

To elucidate the degradation pattern of IGF2BP, the CRC cells with downregulated LINRIS expression were treated with the protein synthesis inhibitor cycloheximide (CHX) and exhibited a shorter IGF2BP2 half-life than the untreated control cells (Fig. 3e).
However, in sh-LINRIS-transfected cells, endogenous IGF2BP2 expression could not increase when cells were treated with the proteasome inhibitor MG132 (Fig. 3f), indicating that the degradation of IGF2BP2 may be linked to a more complex mechanism than the ubiquitin-proteasome pathway.

Apart from the ubiquitin-proteasome system (UBS), cellular proteins could be degraded from the autophagy-lysosome pathway (ALP) after ubiquitination (Fig. 3g) [29]. As shown in Fig. 3h and Additional file 8: Figure S3A, the reduction in the endogenous IGF2BP2 protein was successfully reversed by the autophagy inhibitors bafilomycin A1 (Baf A1), $\mathrm{NH}_{4} \mathrm{Cl}$ and 3-methyladenine (3-MA). In contrast, the autophagy activators Earle's balanced salt solution (EBSS) and Rapamycin (Rap) decreased the levels of IGF2BP2 (Fig. 3i). EBSS-treated cells also exhibited an increased colocalization of IGF2BP2 and LC3B (Fig. $3 j$ and Additional file 8: Figure S3B). Furthermore, we used small guide RNAs (sgRNAs) against autophagy-related gene 5 (ATG5) in DLD-1 cells to block the autophagy system (Additional file 8: Figure S3C). As shown in Additional file 8: Figure S3D, the IGF2BP2 protein levels remained stable when LINRIS was inhibited in ATG5-depleted cells. Overall, the above observation suggests that the LINRIS-mediated degradation of IGF2BP2 was realized through the ubiquitinationautophagy pathway.

\section{MYC-mediated glycolysis was influenced by the interaction between LINRIS and IGF2BP2}

Our group is always eager to explore the metabolic alteration in gastrointestinal cancers $[23,30,31]$. As the mRNA of $M Y C$ (mainly referred to as $c-M y c$ ) is recognized by IGF2BP2 as its well-known downstream target [14], we investigated the change in glycolysis during the interaction between LINRIS and IGF2BP2. As shown in Fig. 4a, b and Additional file 9: Figure S4A, its downstream genes mediating glycolysis, including GLUT-1, PKM2 and LDHA, also exhibited decreased levels in accordance with $M Y C$ when LINRIS was knocked down. In addition, the mRNA levels of MYC, GLUT-1, PKM2 and LDHA positively correlated with the LINRIS levels in CRC tissues (Fig. 4c). As shown in Fig. 4d and e, silencing 


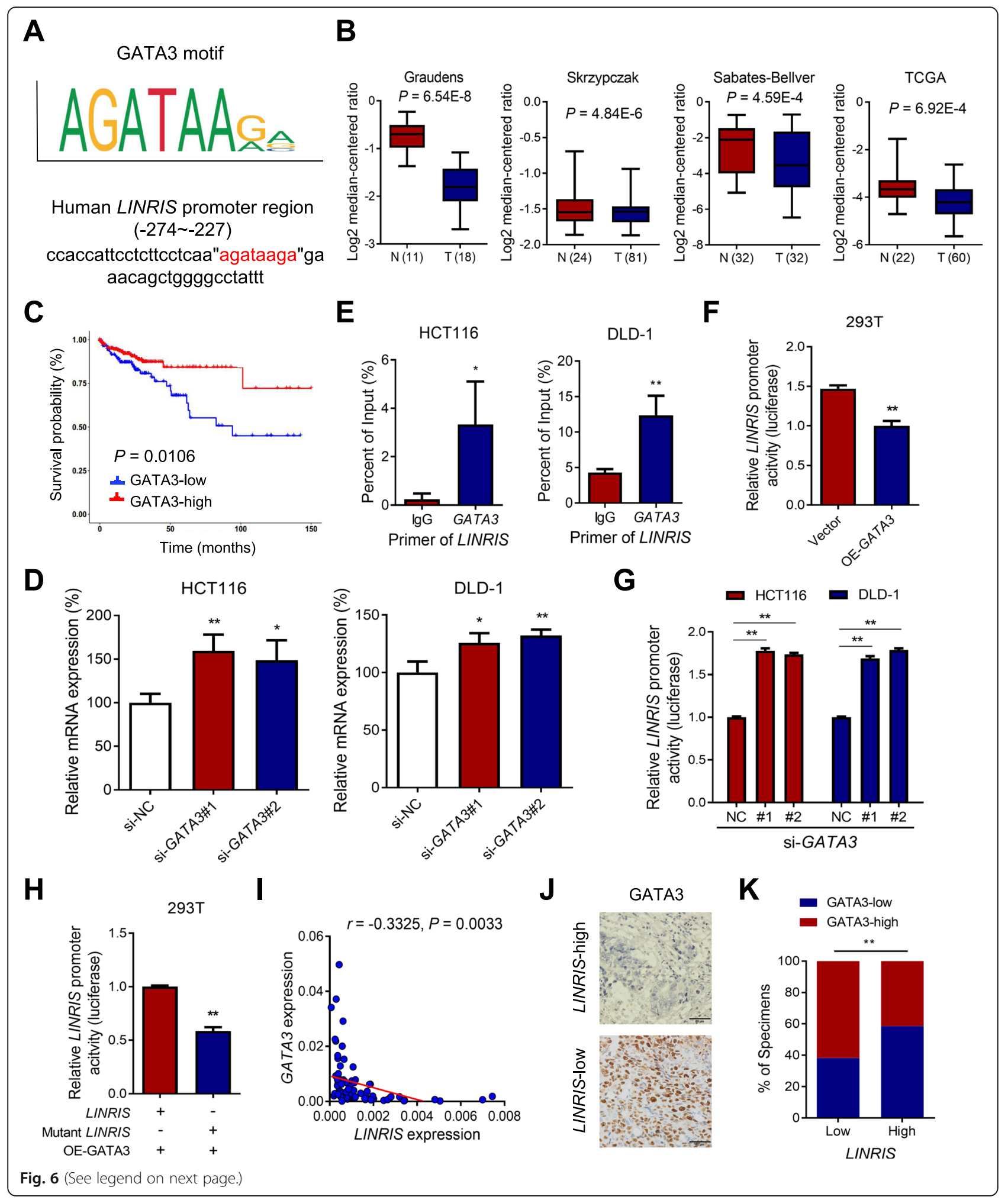




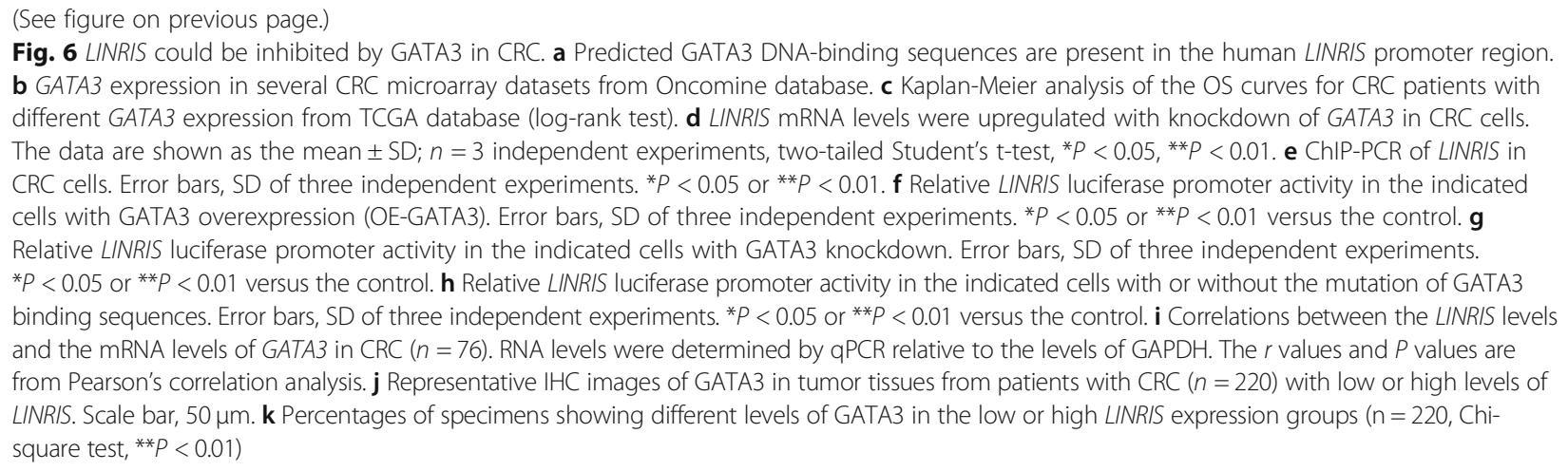

LINRIS resulted in the attenuation of glycolysis in HCT116 and DLD-1 cells. To obtain a deeper view of the metabolic flux of glucose, we incubated CRC cells with ${ }^{13} \mathrm{C}$-labeled glucose before utilizing the liquid chromatography-mass spectrometry (LC-MS). Figure 4f shows that some typical glycolysis productions were reduced with LINRIS knockdown. In conclusion, LINRIS is essential for maintaining glycolysis in cancer cells, which could influence the proliferation of CRC.

Moreover, we transfected CRC cells with plasmids overexpressing IGF2BP2. As shown in Fig. 4g-i and Additional file 9: Figure S4B-S4E, the impaired proliferation and glycolysis induced by LINRIS knockdown was rescued to some extent in HCT116 and DLD-1 cells. In addition, transfecting CRC cells with K139R-mutated IGF2BP2 could fully reverse or even enhance the proliferation impaired by LINRIS (Additional file 9: Figure S4F), further confirming the mechanism of the LINRISIGF2BP2-MYC axis.

\section{Inhibition of LINRIS suppressed CRC growth in vivo}

To investigate whether low LINRIS expression would suppress the growth of CRC tumors in vivo, we injected HCT116 cells with or without LINRIS knockdown (sh-NC, sh-1 and sh-2) into the ceca of BALB/c nude mice. The HCT116/sh-NC cells formed larger and heavier orthotopic tumors in 4 weeks than did the cells with sh-1 or sh-2 (Fig. 5a and $\mathrm{b}$ ).

In addition, we tested the in vivo effect of 'antiLINRIS therapy' with 2 patient-derived xenograft (PDX) models. As shown in Fig. 5c, d and Additional file 10: Figure S5A, inhibition of LINRIS via in vivo-optimized RNA interference (RNAi) significantly suppressed the growth of tumors. Besides, no obvious side effects, such as toxicity or weight loss, were observed (Additional file 10: Figure S5B). IHC staining of the excised tumor sections showed that the expression of Ki-67, which was consistent with that of IGF2BP2 and MYC, decreased with the depletion of LINRIS (Fig. 5e and f). Moreover, we explored the clinical perspective of downregulating LINRIS in combination with chemotherapy as previous reports suggested [32, 33]. In particular, targeting glycolysis has been reported to be a strategy to overcome chemoresistance [34]. As shown in Additional file 10: Figure S5C and S5D, LINRIS inhibition could be performed simultaneously with oxaliplatin treatment. While the proliferation index was decreased, the percentage of apoptotic cells after RNAi and oxaliplatin treatment were higher than the percentage in the control cells (Additional file 10: Figure S5E and S5F). Overall, blocking the LINRIS-IGF2BP2-MYC axis is a promising approach for CRC treatment.

\section{GATA3 inhibited the transcriptional activity of LINRIS}

To investigate what regulates the transcription of LINRIS, we predicted the potential transcription factors with the JASPAR database and found that GATA3 possessed the largest possibility of binding to the promoter of LINRIS (Fig. 6a). CRC microarray data from multiple experiments were analyzed with the Oncomine database, which showed that GATA3 is expressed at lower levels in CRC tissues than in normal tissues (Fig. 6b). Moreover, higher GATA3 expression is linked with a better prognosis of CRC patients according to TCGA database (Fig. 6c). Inhibition of GATA3 successfully emancipated the transcription of LINRIS (Fig. $6 \mathrm{~d}$ and Additional file 11: Figure S6A). The ChIP-PCR assays showed that the LINRIS promoter region were occupied by GATA3 (Fig. 6e), and the luciferase promoter assays showed that GATA3 overexpression inhibited LINRIS activity and vice versa (Fig. $6 f$ and g). Furthermore, the mutation of the GATA3-binding site in the LINRIS promoter abolished this transcriptional regulation (Fig. 6h). By analyzing the mRNA expression of LINRIS and GATA3 in human CRC samples $(n=76)$ from Sun Yat-sen Cancer Center, we also observed a negative correlation between them (Fig. 6i). We also analyzed another 220 samples from 


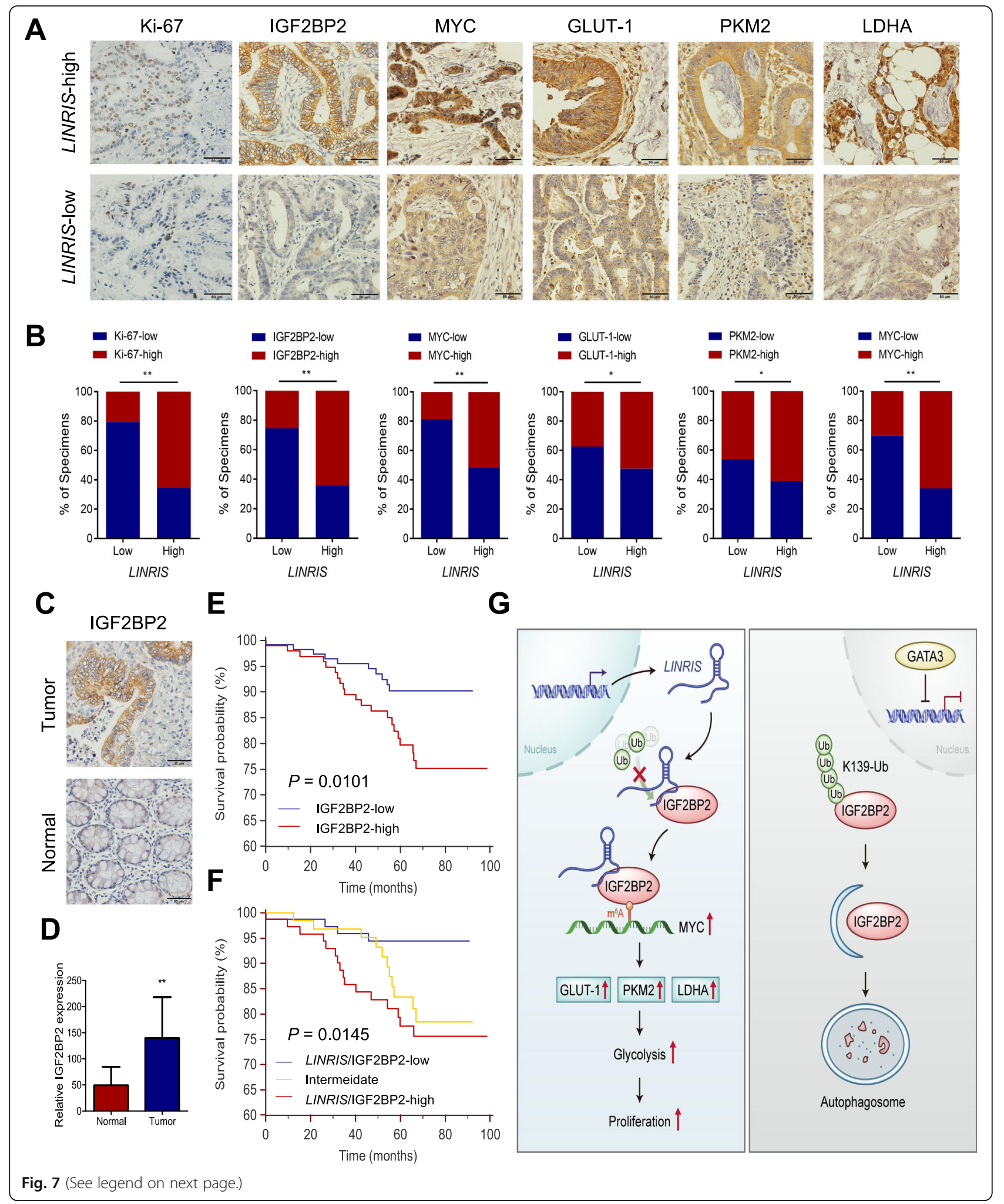


(See figure on previous page.)

Fig. 7 Illustration of LINRIS-IGF2BP2-MYC axis in CRC. a Representative IHC images of Ki-67, IGF2BP2, MYC, GLUT-1 and PKM2 and LDHA in tumor tissues from patients with CRC $(n=220)$ with low or high levels of LINRIS. Scale bar, $50 \mu \mathrm{m}$. b Percentages of specimens showing different levels of Ki-67, IGF2BP2, MYC, GLUT-1 and PKM2 and LDHA in the low or high LINRIS expression groups $(\mathrm{n}=220$, Chi-square test, **P $<0.01)$. $\mathbf{c}$ and $\mathbf{d}$ Representative IHC images (c) and statistical analysis (d) of IGF2BP2 expression in CRC and matched normal tissues. The data are shown as the mean \pm SD; $n=220$, two-tailed Student's $t$-test. e Kaplan-Meier analysis of the OS curves for CRC patients with low $(n=118)$ or high $(n=102)$ IGF2BP2 expression (log-rank test). $\mathbf{f}$ Kaplan-Meier analysis of the OS curves for CRC patients with LINRIS/IGF2BP2-high (both levels of LINRIS and IGF2BP2 were high, $n=76$ ), LINRIS/IGF2BP2-low (both levels of LINRIS and IGF2BP2 were low, $n=76$ ) or intermediate $(n=68)$ expression (log-rank test). $\mathbf{g}$ Proposed working model of this study. LINRIS stabilized IGF2BP2 by binding to its K139 ubiquitination site and subsequently maintained the MYC-induced glycolysis and proliferation of CRC cells. Otherwise, inhibition of LINRIS, such as by GATA3, resulted in more degradation of IGF2BP2 through the ubiquitination-autophagy pathway.

patients with CRC (clinicopathological features are listed in Additional file 12: Table S5) by measuring the expression of LINRIS by RNAScope ${ }^{\circledast}$ ISH assays and that of GATA3 with IHC assays (Fig. 6j). As shown in Fig. 6k, increased LINRIS expression was more likely accompanied by decreased GATA3 expression and vice versa. In conclusion, GATA3 suppressed the transcription of LINRIS.

\section{The LINRIS-IGF2BP2-MYC axis was deeply correlated with the development of CRC}

To further illustrate the clinical and pathological significance of the LINRIS-IGF2BP2-MYC axis in CRC development, we analyzed 220 samples from the patients described above (Additional file 12: Table S5) by measuring the expression of LINRIS by RNAScope ${ }^{\oplus}$ ISH assays, and that of Ki-67, IGF2BP2, MYC, GLUT-1, PKM2 and LDHA with IHC assays (Fig. 7a). As shown in Fig. 7b, the LINRIS-high group was consistent with higher expression of Ki-67, IGF2BP2, MYC, GLUT-1, PKM2 and LDHA, whereas LINRIS-low group exhibited the opposite outcome.

Moreover, IGF2BP2 expression was significantly higher in the tumor tissues from these patients compared with the matched normal tissues (Fig. 7c and d). Higher IGF2BP2 expression was also associated with a poor prognosis for patients with CRC (Fig. 7e). Then we established a combination scoring system separating the tissues into three groups, namely LINRIS/IGF2BP2-high, LINRIS/AGF2BP2-low and intermediate. As expected, the LINRIS/GGFBP2-high group showed a poorer prognosis than the other two groups (Fig. 7f, clinicopathological features are listed in Additional file 13: Table S6). In summary, the LINRIS-IGF2BP2-MYC axis deeply influenced the development and prognosis of CRC and acts as a potential therapeutic target.

\section{Discussion}

Epigenetic regulation is deeply involved in the genesis and development of cancer cells [35-37]. Among the complex regulatory networks, lncRNAs play a crucial role in affecting the fate of tumors [38-41]. In this study, we used RNA-seq to compare advanced tumors with their paired adjacent normal tissues, and we discovered that LINRIS is a highly expressed oncogenic lncRNA that was related to the poor prognosis of patients with CRC. LINRIS is located at chromosome 16q21, and few studies have investigated its function or molecular mechanism. We found that downregulating the expression of LINRIS resulted in the inhibition of CRC cell proliferation. Moreover, we observed that LINRIS interacted with IGF2BP2, whose protein levels were positively correlated with LINRIS expression. IGF2BPs recognize $\mathrm{m}^{6} \mathrm{~A}$-modified mRNAs via $\mathrm{KH}$ domains and maintain their stability by recruiting RNA stabilizers, such as ELAV-like RNA-binding protein 1 (ELAVL1; also known as HuR), matrin 3 (MATR3) and poly (A)-binding protein cytoplasmic 1 (PABPC1) [14, 42]. Therefore, our findings built a bridge between the epigenetic networks of lncRNAs and $\mathrm{m}^{6} \mathrm{~A}$.

Autophagy is a double-edged sword that determines the survival and death of cells under different circumstances, including interacting with lncRNAs [43-45]. By degrading cellular materials, autophagic degradation is able to change the environmental or nutritional conditions and eliminate damaged organelles [46, 47]. In addition, it is associated with ubiquitination, forming a large degradation system instead of an isolated pathway $[29,48]$. In our study, we found that LINRIS bound to a ubiquitination site of IGF2BP2, and this binding blocked IGF2BP2 degradation through the ubiquitination-autophagic pathway. Therefore, its downstream mRNAs including $M Y C$ mRNA were stabilized. To the best of our knowledge, our study is the first to elucidate the degradation pathway of a member of the IGF2BP family.

Because of the Warburg effect, glycolysis is the major method of glucose utilization and determines the progression of cancer cells [49-51]. As MYC mRNA is a typical target of IGF2BP2 and one of the core regulators of glycolysis $[14,52,53]$, we detected the expression of $M Y C$ and its downstream enzymes. The downregulation of MYC-related metabolic enzymes resulted in a reduction in glycolysis, which could also account for the proliferation arrest following LINRIS knockdown. In contrast, the suppression of cancer cell progression and glycolysis could be reversed by overexpressing IGF2BP2, especially by the K139R mutant without the LINRIS-binding site.

GATA3 has been discovered to be linked with the development and invasion of cancer cells [54-56]. In this study, we identified GATA3 as a tumor suppressor gene that interacts with LINRIS. A decreased GATA3 level was 
accompanied by upregulation of LINRIS and a better prognosis for CRC patients. In contrast, overexpression of GATA3 downregulated the transcriptional activity of LINRIS in CRC cells.

Furthermore, the in vivo experiments further identified the antitumor effects of inhibiting LINRIS in CRC, and the analysis of LINRIS/IGF2BP2 expression in the tissues from patients indicated their unique role in the development of CRC; all of these experimental results confirmed the therapeutic potential of targeting the LINRIS-IGF2BP2-MYC axis.

\section{Conclusion}

In conclusion, without inhibition factors such as GATA3, LINRIS binds to the K139 ubiquitination site of IGF2BP2 and prevents it from degradation via the ALP, maintaining the MYC-mediated glycolysis and the proliferation of CRC cells (Fig. $7 \mathrm{~g}$ ).

\section{Supplementary information}

Supplementary information accompanies this paper at https://doi.org/10. 1186/s12943-019-1105-0.

Additional file 1: Table S1. List of reagents and antibodies.

Additional file 2: Table S2. The sequences of siRNAs or shRNAs used in this article.

Additional file 3. Supplementary methods.

Additional file 4: Figure S1, related to Fig. 1. Identification of LINRIS as an oncogenic IncRNA in CRC. (A) Volcano plots of downregulated and upregulated IncRNA, including LINRIS, based on RNA-seq. (B) LINRIS was highly expressed in CRC tissues $(T, n=21)$ compared with the expression in normal colon tissues (N). ${ }^{*} P<0.05,{ }^{* *} P<0.01$. (C) RNA-seq analysis shows the expression of LINRIS transcripts in CRC tissues. (D) In vitrotranscribed LINRIS was reverse transcribed and analyzed with GPCR. The standard curve shows that the $C T$ values decreased linearly with increasing LINRIS copy number. (E) The copy number per cell of LINRIS in CRC cell lines compared with CCD841 based on the standard curve of LINRIS copy number. The data are shown as the mean \pm SD; $n=3$ independent experiments, two-tailed Student's t-test, ${ }^{*} P<0.05,{ }^{* *} P<0.01$. (F) qPCR detection shows the relative RNA levels (left panel) and the copy number per cell (right panel) of LINRIS in 8 human CRC samples (P1-P8). The data are shown as the mean \pm SD; $n=3$ independent experiments. (G) qPCR detection shows the inhibition of LINRIS by shRNAs in the indicated cells. The data are shown as the mean $\pm S D ; n=3$ independent experiments, two-tailed Student's t-test, ${ }^{*} P<0.05$, ${ }^{*} P<0.01$. (H) BrdU assays of the indicated cells with LINRIS knockdown by shRNAs compared with the control. The data are shown as the mean $\pm S D ; n=3$ independent experiments, two-tailed Student's t-test, ${ }^{*} P<0.05$, ${ }^{* *} P<0.01$. (I) FISH assays identifying the subcellular location of LINRIS in DLD-1 cells. Scale bar, $100 \mu \mathrm{m}$.

Additional file 5: Table S3. Correlation between LINRIS expression and clinicopathological features in 118 CRC patients.

Additional file 6: Table S4. Effect of factors on OS in the CRC patients in the univariate and multivariate Cox regression model.

Additional file 7: Figure S2, related to Fig. 2. LINRIS was associated with IGF2BP2 in CRC. (A) The protein score graph of the 18 proteins identified by the RNA pull-down and LC-MS. (B) The emPAI graph of the 18 proteins identified by RNA pull-down assays and LC-MS. (C) Protein cover graph of the 18 proteins identified by RNA pull-down assays and LC-MS. (D) Immunofluorescence assays identifying the subcellular location of IGF2BP2 in the indicated cells. Bar scale: $50 \mu \mathrm{m}$. (E) Computational secondary structure of LINRIS predicted with RNAfold. (F) In vitro- synthesized full-length (FL) and 450-640 nt fragments of LINRIS were incubated with protein lysates from HCT116 cells. RNA pull-down and Western blotting assays were then performed. The data shown represent three independent experiments. (G) qPCR detection of LINRIS levels in 11 CRC cell lines. The data are shown as the mean $\pm S D ; n=3$ independent experiments, two-tailed Student's t-test, ${ }^{*} P<0.05$, ${ }^{* *} P<0.01$. (H) The expression of four representative mRNAs regulated by IGF2BP2 in HCT116 cells with or without sh-LINRIS. The data are shown as the mean $\pm S D$; $\mathrm{n}=3$ independent experiments, two-tailed Student's t-test, ${ }^{*} P<0.05$, ${ }^{* *} P<0.01$.

Additional file 8: Figure S3, related to Fig. 3. LINRIS is involved in the autophagic degradation of IGF2BP2. (A) Western blotting shows the levels of IGF2BP2 in the indicated cells with the knockdown of LINRIS after treatment with or without 3-MA $(10 \mathrm{mg} / \mathrm{ml})$ for $24 \mathrm{~h}$. GAPDH was used as the loading control. (B) Confocal microscopy of HCT116 cells treated with or without EBSS for $3 \mathrm{~h}$. Scale bar, $50 \mu \mathrm{m}$. (C) Western blotting shows the knockout of ATG5 in DLD-1 cells with sgRNA (sg-ATG5) compared with that in control cells. GAPDH was used as the loading control. (D) DLD-1 cells with the knockout of ATG5 and control cells were transfected with shRNAs specific for LINRIS. Cell lysates were analyzed by immunoblotting with GAPDH as the loading control.

Additional file 9: Figure S4, related to Fig. 4. The alteration of MYCmediated glycolysis under the influence of LINRIS. (A) The mRNA levels of MYC and downstream genes, including GLUT-1, PKM2 and LDHA, when inhibiting LINRIS in DLD-1 cells. The data are shown as the mean $\pm S D$; $\mathrm{n}=3$ independent experiments, two-tailed Student's t-test, ${ }^{*} P<0.05$, ${ }^{*} P<0.01$. (B) BrdU assay showing that the overexpression (OE) of IGF2BP2 partially rescued the proliferation inhibition of DLD-1 cells with the knockdown of LINRIS. The data are shown as the mean $\pm S D ; n=3$ independent experiments, two-tailed Student's t-test, ${ }^{*} P<0.05$, ${ }^{* *} P<0.01$. (C) The ECAR was detected in DLD-1 cells with or without sh-1 and overexpressed IGF2BP2 using an XF Extracellular Flux Analyzer. Glucose, oligomycin and 2-DG were injected sequentially at different time points as indicated. The data shown represent three independent experiments. (D) Overexpression of IGF2BP2 partially reversed the suppression of LINRIS knockdown on glycolytic activity in DLD-1 cells. The data are shown as the mean $\pm S D ; n=3$ independent experiments, two-tailed Student's ttest, ${ }^{*} P<0.05$, ${ }^{*} P<0.01$. (E) BrdU assay showing that the overexpression (OE) of the K139 mutant of IGF2BP2 completely rescued the proliferation inhibition of the indicated cells with knockdown of LINRIS. The data are shown as the mean $\pm \mathrm{SD} ; \mathrm{n}=3$ independent experiments, two-tailed Student's t-test, ${ }^{*} P<0.05,{ }^{* *} P<0.01$.

Additional file 10: Figure S5, related to Fig. 5. In vivo experiments elucidated the effect of the inhibition of LINRIS in CRC. (A) qPCR detection shows the relative RNA levels of LINRIS in tumors in two PDX experiments. Error bars, SD of four independent experiments. ${ }^{*} P<0.05$ or ${ }^{*} P<0.01$ versus the control. (B) Curves of the weights of mice treated with RNAi targeting LINRIS in two PDX experiments. (C and D) The volume growth curves of tumors (C) and the tumor weights (D) of PDX\#3 are shown. Ctrl, control. Oxa, oxaliplatin. Error bars, SD of five independent experiments. ${ }^{*} P<0.05$ or ${ }^{*} P<0.01$ versus the control. (E) Representative images of $\mathrm{H} \& \mathrm{E}$ staining, immunohistochemistry staining of Ki-67 and TUNEL from the tumor sections. Scale bar, $100 \mu \mathrm{m}$. (F) Quantification of the proliferation index (Ki-67 proportion) and apoptotic index (TUNEL proportion) in the tumor sections. ${ }^{*} P<0.05$, ${ }^{*} P<0.01$.

Additional file 11: Figure S6, related to Fig. 6. LINRIS could be inhibited by GATA3 in CRC. (A) qPCR detection shows the inhibition of GATA3 by siRNA in the indicated cells. The data are shown as the mean $\pm S D ; n=3$ independent experiments, two-tailed Student's t-test, ${ }^{*} P<0.05$, ${ }^{*} P<0.01$.

Additional file 12: Table S5. Correlation between IGF2BP2 expression and clinicopathological features in 220 CRC patients.

Additional file 13: Table S6. Correlation between LINRIS/IGF2BP2 expression and clinicopathological features in 220 CRC patients.

\section{Abbreviations}

3-MA: 3-methyladenine; ALP: Autophagy-lysosome pathway; Baf A1: Bafilomycin A1; ChIP: Chromatin immunoprecipitation; 
CHX: Cycloheximide; CRC: Colorectal cancer; EBSS: Earle's balanced salt solution; ECAR: Extracellular acidification rate; emPAl: Exponentially modified protein abundance index; ESCC: Esophageal squamous cell carcinoma; FISH: Fluorescence in situ hybridization; GC: Gastric cancer; H\&E: Hematoxylin and eosin; IGF2BP: Insulin-like growth factor 2 mRNA-binding protein; IHC: Immunohistochemically; IP: Immunoprecipitation; KH: K homology; LINRIS: Long Intergenic Non-coding RNA for IGF2BP2 Stability; IncRNAs: Long noncoding RNAs; $\mathrm{m}^{6} \mathrm{~A}: \mathrm{N}^{6}$-methyladenosine; MCP: MS2 coat protein; MS: Mass spectrometry; OS: Overall survival; PDAC: Pancreas ductal adenocarcinoma; PDX: Patient-derived xenograft; qPCR: Quantitative realtime PCR; Rap: Rapamycin; RIP: RNA immunoprecipitation; RNAi: RNA interference; RNA-seq: RNA sequencing; RRMs: RNA recognition motifs; shRNAs: Short hairpin RNAs; siRNAs: Small interfering RNAs; TCGA: The Cancer Genome Atlas; TUNEL: TdT-mediated dUTP nick end labeling; UBS: Ubiquitin-proteasome system; WT: Wild-type; YTHDFs: YT521-B homology domain-containing proteins

\section{Acknowledgments}

We thank doctor Zhi-Ling Li from Sun Yat-sen University Cancer Center for their advice and assistance in the investigation of IGF2BP2 degradation.

\section{Authors' contributions}

Conception and design: YW, Q-NW, H-QJ and R-HX; Development of methodology: YW, J-HL, Q-NW, H-QJ, YJ and R-HX; Acquisition of data (provided animals, acquired and managed patients, provided facilities, etc.): $Y W, J-H L$, Q-NW, YJ, D-SW, JL, X-JL, QM, Y-NW; Analysis and interpretation of data (e.g., statistical analysis, biostatistics, computational analysis): YW, J-HL, Y-XC, QZ and Z-XL; Writing, review, and/or revision of the manuscript: $Y W, R-H X$ and $\mathrm{H}-\mathrm{QJ}$; Administrative, technical, or material support (i.e., reporting or organizing data, constructing databases): YW, J-HL, H-YP, P-SH and Z-LZ; Study supervision: R-HX and H-QJ. Suggestions: RD and X-FZ. All authors read and approved the final manuscript.

\section{Funding}

This research was supported by National Natural Science Foundation of China (81930065, 81871951, 81802438); Natural Science Foundation of Guangdong Province (2014A030312015); Science and Technology Program of Guangdong (2019B020227002); Science and Technology Program of Guangzhou (201904020046, 201803040019, 201704020228); CAMS Innovation Fund for Medical Sciences (2019-12M-5-036) and Pearl River S\&T Nova Program of Guangzhou (201806010002).

\section{Availability of data and materials}

The datasets used and/or analyzed during the current study are available from the corresponding author on reasonable request.

\section{Ethics approval and consent to participate}

The clinical CRC specimens were conducted with the permission by the Institutional Research Ethics Committee of Sun Yat-sen University Cancer Center, China. All animal experiments were performed in accordance with a protocol approved by the Ethics Committee of the Institutional Animal Care of Sun Yat-sen University Cancer Center, China.

\section{Consent for publication}

The content of this manuscript has not been previously published and is not under consideration for publication elsewhere.

\section{Competing interests}

The authors declare that they have no competing interests.

\section{Author details}

${ }^{1}$ State Key Laboratory of Oncology in South China, Collaborative Innovation Center for Cancer Medicine, Sun Yat-sen University Cancer Center, Guangzhou, China. ${ }^{2}$ Department of Medical Oncology, Sun Yat-sen University Cancer Center, Guangzhou, China. ${ }^{3}$ Department of Anatomical and Cellular Pathology, State Key Laboratory of Translational Oncology, Prince of Wales Hospital, The Chinese University of Hong Kong, Hong Kong, China. ${ }^{4}$ Department of Pathology, Sun Yat-sen University Cancer Center, Guangzhou, China. ${ }^{5}$ Precision Diagnosis and Treatment for Gastrointestinal Cancer, Chinese Academy of Medical Sciences, Guangzhou, China.
Received: 4 September 2019 Accepted: 12 November 2019 Published online: 02 December 2019

\section{References}

1. Siegel RL, Miller KD, Jemal A. Cancer statistics, 2019. CA Cancer J Clin. 2018;69:7-34.

2. Zheng RS, Sun KX, Zhang SW, Zeng HM, Zou XN, Chen R, Gu XY, Wei WW, He J. Report of cancer epidemiology in China, 2015. Zhonghua Zhong Liu Za Zhi. 2019;41:19-28.

3. Cabili MN, Trapnell C, Goff L, Koziol M, Tazon-Vega B, Regev A, Rinn JL. Integrative annotation of human large intergenic noncoding RNAs reveals global properties and specific subclasses. Genes Dev. 2011;25:1915-27.

4. Carpenter S, Aiello D, Atianand MK, Ricci EP, Gandhi P, Hall LL, Byron M, Monks B, Henry-Bezy M, Lawrence JB, et al. A long noncoding RNA mediates both activation and repression of immune response genes. Science. 2013;341:789-92.

5. Lee S, Kopp F, Chang TC, Sataluri A, Chen B, Sivakumar S, Yu H, Xie Y, Mendell JT. Noncoding RNA NORAD regulates genomic stability by sequestering PUMILIO proteins. Cell. 2016;164:69-80.

6. Hansen TB, Jensen TI, Clausen BH, Bramsen JB, Finsen B, Damgaard CK, Kjems J. Natural RNA circles function as efficient microRNA sponges. Nature. 2013:495:384-8

7. Zheng J, Huang X, Tan W, Yu D, Du Z, Chang J, Wei L, Han Y, Wang C, Che $X$, et al. Pancreatic cancer risk variant in LINC00673 creates a miR-1231 binding site and interferes with PTPN11 degradation. Nat Genet. 2016:48:747-57.

8. Gupta RA, Shah N, Wang KC, Kim J, Horlings HM, Wong DJ, Tsai MC, Hung $T$, Argani $P$, Rinn $J$, et al. Long non-coding RNA HOTAIR reprograms chromatin state to promote cancer metastasis. Nature. 2010;464:1071-6.

9. Li W, Zheng J, Deng J, You Y, Wu H, Li N, Lu J, Zhou Y. Increased levels of the long intergenic non-protein coding RNA POU3F3 promote DNA methylation in esophageal squamous cell carcinoma cells. Gastroenterology. 2014:146:1714-26.

10. Law JA, Jacobsen SE. Establishing, maintaining and modifying DNA methylation patterns in plants and animals. Nat Rev Genet. 2010;11:204-20.

11. Liu J, Yue Y, Han D, Wang X, Fu Y, Zhang L, Jia G, Yu M, Lu Z, Deng X, et al. A METTL3-METTL14 complex mediates mammalian nuclear RNA N6adenosine methylation. Nat Chem Biol. 2014;10:93-5.

12. Jia G, Fu Y, Zhao X, Dai Q, Zheng G, Yang Y, Yi C, Lindahl T, Pan T, Yang YG, He C. N6-methyladenosine in nuclear RNA is a major substrate of the obesity-associated FTO. Nat Chem Biol. 2011;7:885-7.

13. Wang X, Lu Z, Gomez A, Hon GC, Yue Y, Han D, Fu Y, Parisien M, Dai Q, Jia $\mathrm{G}$, et al. N6-methyladenosine-dependent regulation of messenger RNA stability. Nature. 2014;505:117-20

14. Huang $H$, Weng $H$, Sun W, Qin X, Shi H, Wu H, Zhao BS, Mesquita A, Liu C, Yuan $\mathrm{CL}$, et al. Recognition of RNA N(6)-methyladenosine by IGF2BP proteins enhances mRNA stability and translation. Nat Cell Biol. 2018:20:285-95

15. Xiao W, Adhikari S, Dahal U, Chen YS, Hao YJ, Sun BF, Sun HY, Li A, Ping XL, Lai WY, et al. Nuclear $m(6)$ a reader YTHDC1 regulates mRNA splicing. Mol Cell. 2016;61:507-19.

16. Du H, Zhao Y, He J, Zhang Y, Xi H, Liu M, Ma J, Wu L. YTHDF2 destabilizes $\mathrm{m}(6) \mathrm{A}$-containing RNA through direct recruitment of the CCR4-NOT deadenylase complex. Nat Commun. 2016;7:12626.

17. Bell JL, Wachter K, Muhleck B, Pazaitis N, Kohn M, Lederer M, Huttelmaier S. Insulin-like growth factor 2 mRNA-binding proteins (IGF2BPs): posttranscriptional drivers of cancer progression? Cell Mol Life Sci. 2013;70:2657-75.

18. Li Z, Zhang J, Liu X, Li S, Wang Q, Di Chen HZ, Yu T, Ding J, Li J, et al. The LINC01138 drives malignancies via activating arginine methyltransferase 5 in hepatocellular carcinoma. Nat Commun. 2018;9:1572.

19. Dobin A, Davis CA, Schlesinger F, Drenkow J, Zaleski C, Jha S, Batut P, Chaisson M, Gingeras TR. STAR: ultrafast universal RNA-seq aligner. Bioinformatics. 2013;29:15-21.

20. Li B, Dewey CN. RSEM: accurate transcript quantification from RNA-Seq data with or without a reference genome. Bmc Bioinformatics. 2011;12:323.

21. Love MI, Huber W, Anders S. Moderated estimation of fold change and dispersion for RNA-seq data with DESeq2. Genome Biol. 2014;15:550.

22. Yamamoto $T$, Takano $N$, Ishiwata $K$, Ohmura $M$, Nagahata $Y$, Matsuura $T$, Kamata A, Sakamoto K, Nakanishi T, Kubo A, et al. Reduced methylation of 
PFKFB3 in cancer cells shunts glucose towards the pentose phosphate pathway. Nat Commun. 2014;5:3480.

23. Ju HQ, Lu YX, Chen DL, Zuo ZX, Liu ZX, Wu QN, Mo HY, Wang ZX, Wang DS, Pu HY, et al. Modulation of redox homeostasis by inhibition of MTHFD2 in colorectal Cancer: mechanisms and therapeutic implications. J Natl Cancer Inst. 2018. https://doi.org/10.1093/jnci/djy160.

24. Li S, Zhuang Z, Wu T, Lin JC, Liu ZX, Zhou LF, Dai T, Lu L, Ju HQ. Nicotinamide nucleotide transhydrogenase-mediated redox homeostasis promotes tumor growth and metastasis in gastric cancer. Redox Biol. 2018; 18:246-55.

25. Rupaimoole R, Lee J, Haemmerle M, Ling H, Previs RA, Pradeep S, Wu SY, Ivan C, Ferracin M, Dennison JB, et al. Long noncoding RNA Ceruloplasmin promotes cancer growth by altering glycolysis. Cell Rep. 2015;13:2395-402.

26. Lin A, Li C, Xing Z, Hu Q, Liang K, Han L, Wang C, Hawke DH, Wang S, Zhang $Y$, et al. The LINK-A IncRNA activates normoxic HIF1alpha signalling in triple-negative breast cancer. Nat Cell Biol. 2016;18:213-24.

27. Gruber AR, Bernhart SH, Lorenz R. The ViennaRNA web services. Methods Mol Biol. 2015;1269:307-26.

28. Liu Z, Wang Y, Gao T, Pan Z, Cheng H, Yang Q, Cheng Z, Guo A, Ren J, Xue Y. CPLM: a database of protein lysine modifications. Nucleic Acids Res. 2014; 42:D531-6.

29. Ji CH, Kwon YT. Crosstalk and interplay between the ubiquitin-proteasome system and autophagy. Mol Cells. 2017:40:441-9.

30. Ju HQ, Ying H, Tian T, Ling J, Fu J, Lu Y, Wu M, Yang L, Achreja A, Chen G, et al. Mutant Kras- and p16-regulated NOX4 activation overcomes metabolic checkpoints in development of pancreatic ductal adenocarcinoma. Nat Commun. 2017;8:14437.

31. Wang YN, Zeng ZL, Lu J, Wang Y, Liu ZX, He MM, Zhao Q, Wang ZX, Li T, Lu YX, et al. CPT1A-mediated fatty acid oxidation promotes colorectal cancer cell metastasis by inhibiting anoikis. Oncogene. 2018;37:6025-40.

32. Li Y, Fu L, Li J, Qin Y, Zeng T, Zhou J, Zeng Z, Chen J, Cao T, Ban X, et al. Increased expression of EIF5A2, via hypoxia or gene amplification, contributes to metastasis and angiogenesis of esophageal squamous cell carcinoma. Gastroenterology. 2014;146:1701-13.

33. Liao YJ, Bai HY, Li ZH, Zou J, Chen JW, Zheng F, Zhang JX, Mai SJ, Zeng MS, Sun HD, et al. Longikaurin a, a natural ent-kaurane, induces G2/M phase arrest via downregulation of Skp2 and apoptosis induction through ROS/JNK/c-Jun pathway in hepatocellular carcinoma cells. Cell Death Dis. 2014:5:e1137.

34. Xu RH, Pelicano H, Zhou Y, Carew JS, Feng L, Bhalla KN, Keating MJ, Huang P. Inhibition of glycolysis in cancer cells: a novel strategy to overcome drug resistance associated with mitochondrial respiratory defect and hypoxia. Cancer Res. 2005;65:613-21.

35. Flavahan WA, Gaskell E, Bernstein BE. Epigenetic plasticity and the hallmarks of cancer. Science. 2017;357(6348). https://doi.org/10.1126/science.aal2380.

36. Mohammad HP, Barbash O, Creasy CL. Targeting epigenetic modifications in cancer therapy: erasing the roadmap to cancer. Nat Med. 2019;25:403-18.

37. Wilson CL, Mann DA, Borthwick LA. Epigenetic reprogramming in liver fibrosis and cancer. Adv Drug Deliv Rev. 2017;121:124-32.

38. Yu W, Gius D, Onyango P, Muldoon-Jacobs K, Karp J, Feinberg AP, Cui H. Epigenetic silencing of tumour suppressor gene p15 by its antisense RNA. Nature. 2008;451:202-6.

39. Guffanti G, Galea S, Yan L, Roberts AL, Solovieff N, Aiello AE, Smoller JW, De Vivo I, Ranu H, Uddin M, et al. Genome-wide association study implicates a novel RNA gene, the lincRNA AC068718.1, as a risk factor for post-traumatic stress disorder in women. Psychoneuroendocrino. 2013;38:3029-38.

40. Hu X, Feng Y, Zhang D, Zhao SD, Hu Z, Greshock J, Zhang Y, Yang L, Zhong $X$, Wang LP, et al. A functional genomic approach identifies FAL1 as an oncogenic long noncoding RNA that associates with BMI1 and represses p21 expression in cancer. Cancer Cell. 2014;26:344-57.

41. Peng Z, Liu C, Wu M. New insights into long noncoding RNAs and their roles in glioma. Mol Cancer. 2018;17:61.

42. Noubissi FK, Elcheva I, Bhatia N, Shakoori A, Ougolkov A, Liu J, Minamoto T, Ross J, Fuchs SY, Spiegelman VS. CRD-BP mediates stabilization of betaTrCP1 and c-myc mRNA in response to beta-catenin signalling. Nature. 2006:441:898-901.

43. Shintani T, Klionsky DJ. Autophagy in health and disease: a double-edged sword. Science. 2004;306:990-5.

44. Cai Q, Wang S, Jin L, Weng M, Zhou D, Wang J, Tang Z, Quan Z. Long noncoding RNA GBCDRInc1 induces chemoresistance of gallbladder cancer cells by activating autophagy. Mol Cancer. 2019;18:82.
45. YiRen $H$, YingCong $Y$, Sunwu $Y$, Keqin $L$, Xiaochun $T$, Senrui $C$, Ende $C$, XiZhou L, Yanfan C. Long noncoding RNA MALAT1 regulates autophagy associated chemoresistance via miR-23b-3p sequestration in gastric cancer. Mol Cancer. 2017:16:174.

46. Mizushima N, Komatsu M. Autophagy: renovation of cells and tissues. Cell. 2011;147:728-41.

47. Madrigal-Matute J, Cuervo AM. Regulation of liver metabolism by autophagy. Gastroenterology. 2016;150:328-39.

48. Jiao L, Zhang HL, Li DD, Yang KL, Tang J, Li X, Ji J, Yu Y, Wu RY, Ravichandran $\mathrm{S}$, et al. Regulation of glycolytic metabolism by autophagy in liver cancer involves selective autophagic degradation of HK2 (hexokinase 2). Autophagy. 2018;14:671-84.

49. Gogvadze V, Zhivotovsky B, Orrenius S. The Warburg effect and mitochondrial stability in cancer cells. Mol Aspects Med. 2010;31:60-74.

50. Liberti MV, Locasale JW. The Warburg effect: how does it benefit Cancer cells? Trends Biochem Sci. 2016:41:211-8.

51. Goncalves MD, Cantley LC. A glycolysis outsider steps into the cancer spotlight. Cell Metab. 2018;28:3-4.

52. Tateishi K, lafrate AJ, Ho Q, Curry WT, Batchelor TT, Flaherty KT, Onozato ML, Lelic N, Sundaram S, Cahill DP, et al. Myc-driven glycolysis is a therapeutic target in Glioblastoma. Clin Cancer Res. 2016;22:4452-65.

53. Hsieh AL, Walton ZE, Altman BJ, Stine ZE, Dang CV. MYC and metabolism on the path to cancer. Semin Cell Dev Biol. 2015;43:11-21.

54. Sidibe A, Ropraz P, Jemelin S, Emre Y, Poittevin M, Pocard M, Bradfield PF, Imhof BA. Angiogenic factor-driven inflammation promotes extravasation of human proangiogenic monocytes to tumours. Nat Commun. 2018:9:355.

55. Lin MC, Lin JJ, Hsu CL, Juan HF, Lou PJ, Huang MC. GATA3 interacts with and stabilizes HIF-1alpha to enhance cancer cell invasiveness. Oncogene. 2017;36:4380.

56. Bertucci F, Ng C, Patsouris A, Droin N, Piscuoglio S, Carbuccia N, Soria JC, Dien AT, Adnani Y, Kamal M, et al. Genomic characterization of metastatic breast cancers. Nature. 2019;569:560-4.

\section{Publisher's Note}

Springer Nature remains neutral with regard to jurisdictional claims in published maps and institutional affiliations.

Ready to submit your research? Choose BMC and benefit from:

- fast, convenient online submission

- thorough peer review by experienced researchers in your field

- rapid publication on acceptance

- support for research data, including large and complex data types

- gold Open Access which fosters wider collaboration and increased citations

- maximum visibility for your research: over $100 \mathrm{M}$ website views per year

At $\mathrm{BMC}$, research is always in progress.

Learn more biomedcentral.com/submissions 